\title{
CRIDECO Anticholinergic Load Scale: An Updated Anticholinergic Burden Scale. Comparison with the ACB Scale in Spanish Individuals with Subjective Memory Complaints
}

\author{
Hernán Ramos ${ }^{1,2} \mathbb{D}$, Lucrecia Moreno ${ }^{1,2}{ }^{\mathbb{D}}$, Jordi Pérez-Tur ${ }^{1,3} \mathbb{D}$, Consuelo Cháfer-Pericás $1,4(\mathbb{D}$, \\ Gemma García-Lluch ${ }^{1,4}$ and Juan Pardo 1,5,*D
}

check for

updates

Citation: Ramos, H.; Moreno, L.;

Pérez-Tur, J.; Cháfer-Pericás, C.

García-Lluch, G.; Pardo, J. CRIDECO

Anticholinergic Load Scale: An

Updated Anticholinergic Burden

Scale. Comparison with the ACB

Scale in Spanish Individuals with

Subjective Memory Complaints. J

Pers. Med. 2022, 12, 207. https://

doi.org/10.3390/jpm12020207

Academic Editor: Marco Costanzi

Received: 4 January 2022

Accepted: 31 January 2022

Published: 3 February 2022

Publisher's Note: MDPI stays neutral with regard to jurisdictional claims in published maps and institutional affiliations.

Copyright: (c) 2022 by the authors. Licensee MDPI, Basel, Switzerland. This article is an open access article distributed under the terms and conditions of the Creative Commons Attribution (CC BY) license (https:// creativecommons.org/licenses/by/ $4.0 /)$.
1 Cátedra DeCo MICOF-CEU UCH, Universidad Cardenal Herrera-CEU, CEU Universities, 46115 Valencia, Spain; ramgarher@alumnos.uchceu.es (H.R.); lmoreno@uchceu.es (L.M.); jpereztur@ibv.csic.es (J.P.-T.); m.consuelo.chafer@uv.es (C.C.-P.); gemma.garcia2@alumnos.uchceu.es (G.G.-L.)

2 Department of Pharmacy, Universidad Cardenal Herrera-CEU, CEU Universities, 46115 Valencia, Spain

3 Institut de Biomedicina de València-CSIC, CIBERNED, Unitat Mixta de Neurologia i Genetica, IIS La Fe, 46010 Valencia, Spain

4 Alzheimer Disease Research Group, Health Research Institute La Fe, 46026 Valencia, Spain

5 Embedded Systems and Artificial Intelligence Group, Universidad Cardenal Herrera-CEU, CEU Universities, 46115 Valencia, Spain

* Correspondence: juaparal@uchceu.es

\begin{abstract}
The increase in life expectancy has also been accompanied by an increase in the use of medication to treat chronic diseases. Polypharmacy is associated with medication-related problems such as the increase in the anticholinergic burden. Older people are more susceptible to anticholinergic effects on the central nervous system and this, in turn, may be related to cognitive impairment. In this paper, we develop an updated anticholinergic burden scale, the CRIDECO Anticholinergic Load Scale (CALS) via a systematic review of the literature and compare it with the currently most used Anticholinergic Burden Scale (ACB). Our new scale includes 217 different drugs with anticholinergic properties, 129 more than the ACB. Given the effect that anticholinergic medications have on cognitive performance, we then used both scales to investigate the relationship between anticholinergic burden and cognitive impairment in adult Spanish subjects with subjective memory complaint. In our population, we observed an association between cognitive impairment and the anticholinergic burden when measured by the new CALS, but not when the ACB was applied. The use of a more comprehensive and upgraded scale will allow better discrimination of the risk associated with the use of anticholinergic medications on cognitive impairment. CALS can help raise awareness among clinicians of the problems associated with the use of medications, or combinations of them, with large anticholinergic effect, and promote a better personalized pharmacological approach for each patient.
\end{abstract}

Keywords: cognitive impairment; anticholinergic burden scale; anticholinergic drug; subjective memory complaint

\section{Introduction}

Life expectancy is one of the most important measures for assessing the overall health of a population and data indicate a very positive trend in recent years. Spain is the fourth country with the highest life expectancy after Japan, Switzerland and the Czech Republic [1]. This increase in life expectancy has also been accompanied by an increase in the appearance of age-related chronic diseases and the concomitant use of medications to treat them. This situation is reflected in the increase in polypharmacy in recent years, considering the term polypharmacy as the consumption of five or more medicines [2]. The prevalence of polypharmacy in our country, according to the latest studies, is $27.3 \%$ [2].

Polypharmacy is associated with increased drug-drug interactions, increased risk of adverse drug reactions [3], decreased adherence to treatment [4], increased risk of frailty [5], 
increased risk of hip fractures [6] and increased risk of falls and hospitalizations [7]. In this context, it is important to note that the use of drugs with anticholinergic properties has increased in recent years [8].

Anticholinergic medications are drugs that block the neurotransmitter acetylcholine from binding to muscarinic receptors [9]. The cumulative effect of taking drugs with anticholinergic actions is known as the anticholinergic burden [10]. On the other hand, the effects of anticholinergic drugs are divided into peripheral (dry mouth, blurred vision, constipation, tachycardia and urinary retention) and central (writing confusion, dizziness and even cognitive impairment) [9]. Moreover, older people are more susceptible to anticholinergic effects on the central nervous system due to a higher permeability of the blood-brain barrier [11]. The estimated prevalence of the use of these drugs ranges from $12.5 \%$ [12] to $49.5 \%$ [13] depending on the population and the scale used.

Within the muscarinic receptors, there are different subtypes, M1 to M5. M1 receptors are the most common receptors in the central nervous system and play important roles in executive abilities and episodic memory in the hippocampus and prefrontal cortex [14]. Furthermore, antagonism of M2 (memory processing) and M4 receptors (acetylcholine level regulation) can lead to cognitive disorders and cell death [15]. This leads to a direct link between cognitive impairment (CI) and the use of anticholinergic drugs. Numerous studies associate the use of anticholinergic drugs with CI [16-19], and several longitudinal studies even consider the use of these drugs as a risk factor for dementia [20-23]. In addition, the use of these drugs has been linked to higher mortality [24,25] and increased risk of hospitalizations [26].

However, the existence of many scales measuring the anticholinergic burden means that the conclusions drawn can be variable. Moreover, many of them are more than a decade old, such as the Anticholinergic Drug Scale (ADS) or the Anticholinergic Cognitive Burden (ACB) scale, and continue to obtain the highest percentages in quality today [27]. In addition, the ACB scale is currently the most widely used and includes 88 drugs with an anticholinergic effect [27]. Continued drug approvals by regulatory agencies make it necessary to update these scales, as many now commonly used anticholinergic drugs are not included.

Alzheimer's disease (AD) has now been shown to be a biological continuum between the early asymptomatic (preclinical AD), mildly symptomatic (subjective cognitive decline) or moderately symptomatic (mild cognitive impairment) stages and the most severe phase (dementia) [28]. The term subjective cognitive decline (SCD) was conceived to describe cognitively intact individuals who are concerned about declining cognitive function [29]. In fact, SCD has been classified as the stage prior to mild cognitive impairment (MCI) in individuals with positive AD biomarkers according to the National Institute of Ageing research framework [28]. Over the last decade, knowledge of SCD has increased enormously, positioning it as the earliest symptomatic manifestation of AD [30]. Subjective memory complaint (SMC) is one of the criteria necessary to include patients in SCD, along with a normal score on cognitive tests used to classify MCI or prodromal AD [31]. Furthermore, SMC has been linked to a higher incidence of dementia, doubling the likelihood of developing the pathology [32].

This implies that studies carried out in the population with SMC will not discriminate well between risk and protective factors for CI compared to those studies carried out in the general population. This is the case of anticholinergic drugs, where we hypothesize that the anticholinergic burden scales currently in use, as they only include a part of the existing anticholinergic drugs, may not differentiate well the risk of CI related to the use of these drugs in a special sample.

The aim of this paper is the development of an updated anticholinergic burden scale through a systematic review of the literature and the inclusion of new drugs. In addition, this new scale will be applied to adult Spanish subjects with SMCs, in order to explore the relationship between anticholinergic treatment and CI. 


\section{Materials and Methods}

\subsection{Systematic Review}

A systematic review was conducted in PubMed and Web of Science databases to identify the main anticholinergic scales used following PRISMA guidelines [33]. This review was carried out between 6 and 10 October 2021. The keywords used were: "review" AND "anticholinergic" AND "burden" AND "scale". The search and review process are presented in Figure 1. Papers that did not include anticholinergic burden scales or were not written in English were excluded. Manuscripts that presented lists of drugs with an assigned score were included. A total of 167 records were first identified, 69 in PubMed and 98 in Web of Science. After removing any duplicates, 110 records were selected. For the title-based screening, those that related anticholinergic use to other pathologies different from CI, or did not mention any tool or scale, were eliminated. Of the remaining 45 records, only those that included the use of an anticholinergic scale in their abstract were selected. Thus, sixteen full-text articles were assessed for eligibility.

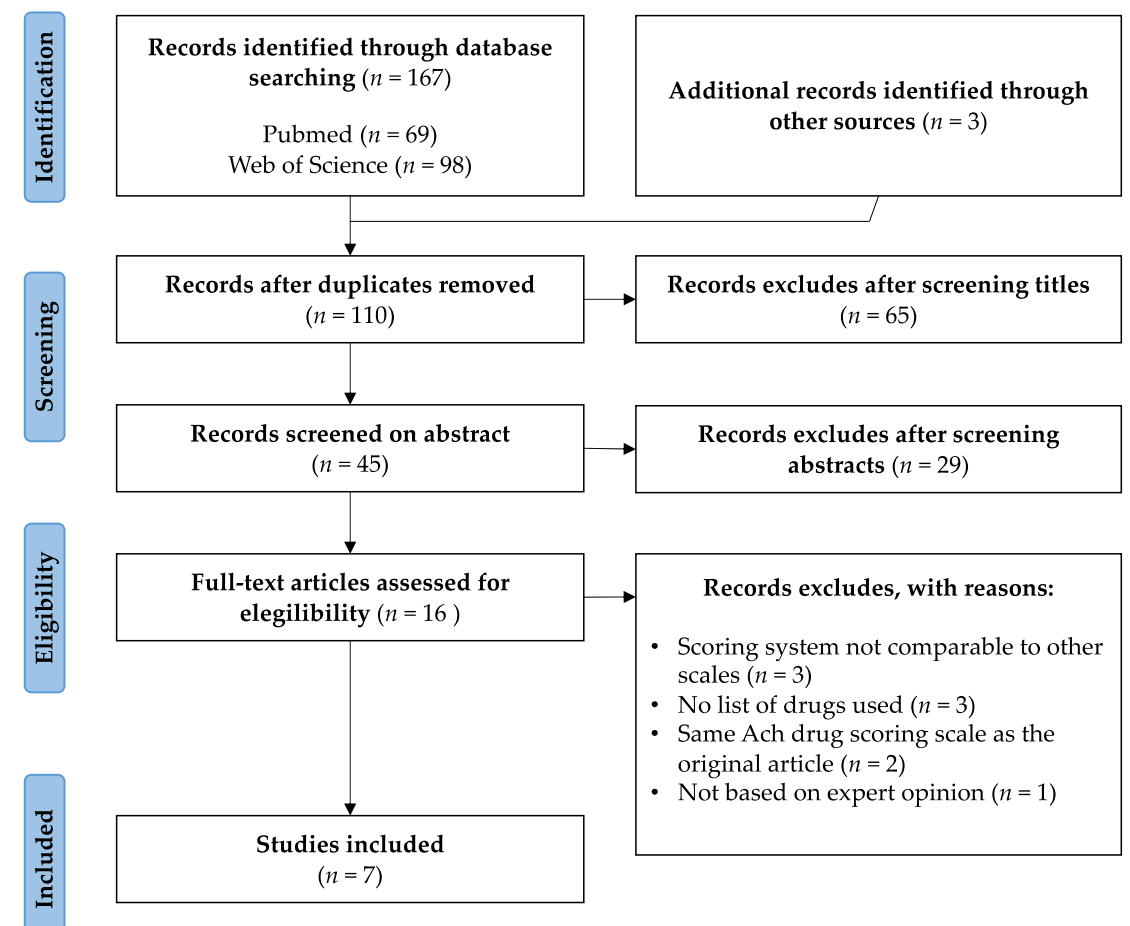

Figure 1. PRISMA flow diagram of study selection.

From them, three manuscripts whose scoring system was not comparable to other scales were excluded [34-36]. Two identified manuscripts used the same scales as those already selected in their original articles and were therefore also excluded $[37,38]$. Finally, three manuscripts that did not include the list of drugs used $[27,39,40]$ and one manuscript not based on expert opinion were also excluded [41].

The final list of manuscripts included seven scales: ADS, Anticholinergic Risk Scale (ARS), ACB, Duran Scale (DS), Salahudeen Scale (SS), German Anticholinergic Burden Scale (GABS) and Korean Anticholinergic Burden Scale (KABS) [42-48].

\subsection{Development of a New Anticholinergic Burden Scale}

All the tools chosen rated from 1 to 3 the anticholinergic score, except the review by Duran et al., which scores the drugs as high potency (3) or low potency (1 or 2) [45]. In this case, we scored from 1 or 2 when in Durán's review the different authors gave both scores, and if the score was homogeneous, we scored directly with the value shown in the corresponding column. The same was true for Salahudeen's review, offering several scores 
for the same drug [46]. Furthermore, in Durán's review, drugs with different scores were classified as discrepant ("Disc").

In the CRIDECO Anticholinergic Load Scale (CALS), we classified the anticholinergic potency of the drugs from 1 (lowest) to 3 (highest). When in the previous scales the score for a particular drug was homogeneous (all of them scored the same drug with the same score) we used such a score. When there were discrepancies between scales, we chose the mean value rounding to the nearest whole number.

The Medicines Database of the Spanish General Council of Pharmaceutical Associations was used to review the medicines commercialized in Spain [49]. Topical, otic, ophthalmic and nasal forms were excluded due to lower systemic absorption [47]. In the event of doubt, these drugs were studied individually, and their final score was determined by the expert committee. This committee was composed of a professor of pharmacology, a clinical pharmacist and a community pharmacist. The pharmacists based their final decisions on their clinical experience and a review of the current literature.

\subsection{Subject Recruitment and Data Collection}

Recruitment was conducted in 19 community pharmacies in the Valencian region (Spain) over 18 months (September 2018 to March 2020). Patient recruitment ended with the onset of the SARS-CoV-2 pandemic in Spain. The protocol used was the same as in previous studies [50,51].

All community pharmacists were trained by the project's neurologists. During the dispensing routine, the pharmacist identified, either by express reference from the patient, by a close relative or by direct observation by the pharmacist, signs of SMC or possible cognitive impairment, depressive feelings, increased sleepiness, impaired speech or object recognition, difficulty in performing complex activities such as using public transport, problems with money management or medical treatment. Subjects who met the inclusion criteria were informed of the study in the community pharmacies. Inclusion criteria were age 50 years or older, SMCs and willingness to participate. Conversely, the exclusion criteria were age less than 50 years, no SMCs, diagnosis of any dementia, severe sensory deficits (blindness, deafness) and physical disability that could interfere with the performance of the tests.

This study was reviewed and approved by the Institutional Review Board (IRB) of Universidad CEU Cardenal Herrera (CEI18/027, date of approval: 2 February 2018) and by the IRB of the Arnau de Vilanova Hospital (MOR-ROY-2018-013, date of approval: 18 July 2018). All subjects gave written informed consent in accordance with the Declaration of Helsinki.

The variables used were collected through a personalized interview at the community pharmacy, which lasted approximately $40 \mathrm{~min}$. Moreover, the questionnaire used included additional lifestyle variables and dietary habits. The Anatomical Therapeutic Chemical (ATC) code of the World Health Organization (WHO) Collaborating Centre for Drug Statistic Methodology was used to classify the drugs [52]. When a combination was identified, it was considered as such, instead of considering active ingredients separately. Nevertheless, an exception was made with 12 ATC codes (N06CA01, N02BE51, N02BA51, H02BX93, R05DA20, M01AE51, R01BA52, R05CA10, C07CB03, R05FA02, N04BA03 and C03EB01). The above-mentioned ATC codes represent several combinations with diverse active ingredients, each of which has a different anticholinergic burden. In this case, patients taking these codes were identified and the combination ATC codes were separated into individual ATC codes.

\subsection{Cognitive Impairment Assessment}

Patients were assessed using three validated tests: Memory Impairment Screen (MIS), Short Portable Mental Questionnaire (SPMSQ) and Semantic Verbal Fluency Test (SVF). The tests used were chosen on the recommendation of the Valencian Society of Neurology. The idea of using these three tests was to detect as many true positives as possible and thus 
increase the accuracy of the overall process. Subjects with positive results in at least one of these tests were classified as possible CI patients. With this protocol, the CI confirmation rate in neurology was $90 \%$ [50].

\subsubsection{Memory Impairment Screen}

The MIS is a brief test of memory disorders using free and selectively facilitated recall of four words, giving each word a value of 2 points when recalled without a cue and a value of 1 when a cue is needed (0-8) [53]. It uses controlled learning and selectively facilitated recall techniques. Controlled learning is based on the subject identifying the word to be remembered according to a semantic cue (category of each word). The same semantic cue is used for facilitated recall. The most effective cut-off score is $\leq 4$ points, where the sensitivity for general dementia in the Spanish population was $74 \%$ and the specificity $96 \%$ [54]. For $\mathrm{AD}$, it obtained sensitivities of $86 \%$ and $96 \%$, respectively [54].

\subsubsection{Short Portable Mental State Questionnaire (Spanish Version)}

The SPMSQ test assesses different aspects of intellectual functioning, including shortterm memory, long-term memory, information about current events, orientation and the ability to perform serial mathematical work [55]. It is a short test that is easy to perform and score, with a total of 10 items and a cut-off point of 3 or more errors, does not require specific equipment and applies to illiterate people. The Spanish version of the test showed high sensitivity and specificity for CI, 85.7\% and 79.3\%, respectively [56].

\subsubsection{Semantic Verbal Fluency}

The SVF test measures the number of items of a category (in this case animals) that a subject can recall in one minute. This test is very specific for temporal lesions, and in fact has been used preferentially in patients with $\mathrm{AD}$, where there is a progressive alteration of semantic memory, attributed to alterations in the frontal and temporal lobes [57]. Not only is it valid for the detection of patients with $\mathrm{AD}$ dementia, but it can also discriminate with high sensitivity between patients with normal cognition, MCI and vascular dementia [58]. The recommended cut-off point is less than 10 points, at which the sensitivity for the detection of general dementia for this test in Spain was $90 \%$ and the specificity $94 \%$ in a population with a poor educational level [59].

\subsection{Statistical Treatment}

A machine learning technique protocol was used in the community pharmacy to rapidly select candidates for further screening via a question-based CI test [60]. A new artificial intelligence tool, developed by the research group, and based on an ensemble of different machine learning supervised techniques (such as bagging and boosting decision trees) was used to calculate the MCI probability result on performing the tests [60]. The idea was to maximize the selection process by attending to those factors that entail a high probability of positive results in the screening tests. For this reason, the subjective memory complaint was used as a criterion for inclusion in the study. Thus, different statistical and artificial intelligence data analysis techniques were used to examine the collected data.

After completion of the follow-up of the subjects, all the information was stored in a database designed specifically for this study. Subsequently, the data were checked by reviewing the subjects' medical records during the overall process and a subsequent data cleansing process was conducted to check the completeness and correctness of the dataset. The statistical analysis was carried out using advanced statistical treatment RStudio@ (version 4.1.1 (10 August 2021) Integrated Development for R. RStudio, PBC, Boston, MA, USA) [61].

Our initial hypothesis was that the new scoring system, the CALS, would discriminate better than the ACB scale CI for individuals. Thus, the sample size to conduct the study was calculated using $G^{*}$ Power statistical software (version 3.1.9.6, Düsseldorf, Germany) [62]. A two-sample t-test was carried out to check differences between two independent means 
(two groups). The parameters were set as a two-sided test with medium effect size, power of 0.95 and a significance level of 0.05 . This concluded a minimum of 210 subjects and our final total sample was 512 subjects.

Moreover, Chi-squared tests were also conducted to discover if $\mathrm{CI}$ is dependent on a relevant (higher than 2) anticholinergic load with the new CALS, and in that sense, a significance level was established at 0.05 .

\section{Results}

\subsection{CRIDECO Anticholinergic Load Scale}

Through our systematic review of the literature, we included seven scales: ADS, ARS, ACB, Duran Scale (DS), Salahudeen Scale (SS), GABS and KABS (Figure 2) [42-48]. Figure 2 shows the selected scales, the number of drugs included in each one, the first author, year of publication and country, together with our new scale. The final CALS included a total of 217 different drugs with anticholinergic properties.

\begin{tabular}{|c|c|c|c|}
\hline \multicolumn{4}{|c|}{ Seven final anticholinergic scales } \\
\hline Abbreviation & Anticholinergic scales & Number of $A C H$ drugs & Study ID \\
\hline ADS & Anticholinergic Drug Scale & 117 & Carnahan 2006 USA \\
\hline ARS & Anticholinergic Risk Scale & 49 & Rudolph 2008 USA \\
\hline $\mathrm{ACB}$ & Anticholergic Cognitive Burden Scale & 88 & Boustani 2008 USA \\
\hline DS & Duran Scale & 100 & Duran 2013 Ecuador \\
\hline SS & Salahudeen Sale & 195 & Salahudeen 2015 New Zealand \\
\hline GABS & German Anticholinergic Burden Scale & 151 & Kiesel 2018 Germany \\
\hline KABS & Korean Anticholinergic Activity Scale & 138 & Kwanghee 2019 Korea \\
\hline CALS & CRIDECO Anticholinergic Load Scale & 217 & This study \\
\hline
\end{tabular}

Figure 2. Summary of the number of anticholinergic $(\mathrm{ACH})$ drugs on each scale.

After our systematic review, we selected 125 low potency (Score 1) anticholinergic drugs (Table 1), 28 drugs with medium potency (Table 2) and 62 drugs with high anticholinergic potency (Table 3). Of these, colchicine was ruled out, as one author rated this drug with a score of 1 or 3 [46], another scale rated it as discrepant (Disc) and two scales explicitly rated it with a null anticholinergic score $[42,48]$.

Table 1. Low potency anticholinergics (Score 1).

\begin{tabular}{|c|c|c|c|c|c|c|c|c|}
\hline Drug Name & ATC Code & $\begin{array}{l}\text { Carnahan } \\
2006\end{array}$ & $\begin{array}{l}\text { Rudolph } \\
2008\end{array}$ & $\begin{array}{c}\text { Boustani } \\
2008\end{array}$ & $\begin{array}{l}\text { Durán } \\
2013\end{array}$ & $\begin{array}{c}\text { Salahudeen } \\
2015\end{array}$ & $\begin{array}{c}\text { Kiesel } \\
2018\end{array}$ & $\begin{array}{l}\text { Jun } \\
2019\end{array}$ \\
\hline Aclidinium inh & R03BB05 & & & & & & 1 & \\
\hline Alimemazine * & R06AD01 & & & 1 & 2 & 1 or 2 & & 1 \\
\hline Alprazolam & N05BA12 & 1 & & 1 & Disc & 1 or 3 & 1 & 1 \\
\hline Alverine & A03AX08 & & & 1 & Disc & 1 or 2 & & 0 \\
\hline Amisulpride & N05AL05 & & & & & & & 1 \\
\hline Ampicillin & J01CA01 & 1 & & & Disc & 1 & 1 & 0 \\
\hline Aripiprazole & N05AX12 & & & & & 1 & 1 & 1 \\
\hline Asenapine & N05AH05 & & & & & 1 & 1 & \\
\hline Atenolol & C07AB03 & 0 & & 1 & & 1 & 1 & 0 \\
\hline Azathioprine & L04AX01 & 1 & & & Disc & 1 & 1 & 0 \\
\hline Benazepril & C09AA07 & 0 & & & Disc & 1 & 1 & 0 \\
\hline Betaxolol & C07AB05 & 0 & & & Disc & 1 & 1 & 0 \\
\hline Bisacodyl & A06AB02 & 0 & & & Disc & 1 & 1 & 0 \\
\hline
\end{tabular}


Table 1. Cont.

\begin{tabular}{|c|c|c|c|c|c|c|c|c|}
\hline Drug Name & ATC Code & $\begin{array}{c}\text { Carnahan } \\
2006\end{array}$ & $\begin{array}{l}\text { Rudolph } \\
2008\end{array}$ & $\begin{array}{c}\text { Boustani } \\
2008\end{array}$ & $\begin{array}{c}\text { Durán } \\
2013\end{array}$ & $\begin{array}{l}\text { Salahudeen } \\
2015\end{array}$ & $\begin{array}{c}\text { Kiesel } \\
2018\end{array}$ & $\begin{array}{l}\text { Jun } \\
2019\end{array}$ \\
\hline Bromocriptine & N04BC01 & 1 & & & 1 or 2 & 1 & 1 & 0 \\
\hline Bromperidol & N05AD06 & & & & & & & 1 \\
\hline Bupropion & N06AX12 & 0 & & 1 & Disc & 1 & 1 & 1 \\
\hline Captopril & C09AA01 & 1 & & 1 & Disc & 1 & 1 & 0 \\
\hline Cefamandole & J01DC03 & 1 & & & Disc & 1 & & 0 \\
\hline Cefoxitin & J01DC01 & 1 & & & Disc & 1 & & 0 \\
\hline Celecoxib & M01AH01 & 0 & & & Disc & 1 & 1 & 0 \\
\hline Cephalothin & J01DB03 & 1 & & & Disc & 1 & & 0 \\
\hline Cetirizine & R06AE07 & 0 & 2 & & 2 & 1 or 2 & 1 & 1 \\
\hline Cinnarizine & N07CA02 & & & & & & & 1 \\
\hline Chlordiazepoxide & N05BA02 & 1 & & & 1 & 1 & 1 & 1 \\
\hline Chlorthalidone & C03BA04 & 1 & & 1 & Disc & 1 & 1 & 0 \\
\hline Citalopram & N06AB04 & 0 & & & 1 & 1 & 1 & 1 \\
\hline Clindamycin & J01FF01 & 1 & & & Disc & 1 & 1 & 0 \\
\hline Clonazepam & N03AE01 & 1 & & & 1 & 1 & 1 & 1 \\
\hline Clorazepate & N05BA05 & 1 & & 1 & Disc & 1 or 3 & 1 & 1 \\
\hline Codeine & R05DA04 & 1 & & 1 & 1 or 2 & 1 or 2 & 1 & 1 \\
\hline Colchicine & M04AC01 & 0 & & & Disc & 1 or 3 & & 0 \\
\hline Cortisone & H02AB10 & 1 & & & Disc & 1 & & \\
\hline Cycloserine & J04AB01 & 1 & & & Disc & 1 & & 0 \\
\hline Cyclosporine & L04AD01 & 1 & & & Disc & 1 & & 0 \\
\hline Desloratadine & R06AX27 & & & & & 1 & 1 & 1 \\
\hline Desvenlafaxine & N06AX23 & & & & & & & 1 \\
\hline Dexamethasone & $\mathrm{H} 02 \mathrm{AB} 02$ & 1 & & & Disc & 1 & 1 & 0 \\
\hline Dextromethorphan & R05DA09 & 0 & & & Disc & 1 & 1 & 1 \\
\hline Diazepam & N05BA01 & 1 & & 1 & 1 & 1 & 1 & 1 \\
\hline Digitoxin & C01AA04 & 1 & & & 1 & 1 & 1 & \\
\hline Digoxin & C01AA05 & 1 & & 1 & Disc & 1 or 3 & 1 & 1 \\
\hline Diltiazem & C08DB01 & 1 & & & Disc & 1 & 1 & 0 \\
\hline Dipyridamole & B01AC07 & 1 & & 1 & Disc & 1 & 1 & 0 \\
\hline Disopyramide & C01BA03 & 2 & & 1 & 2 & 1 or 2 & & \\
\hline Domperidone & A03FA03 & & & & 1 & 1 & 1 & 0 \\
\hline Entacapone & N04BX02 & 0 & 1 & & 1 & 1 & 1 & 0 \\
\hline Escitalopram & N06AB10 & 0 & & & Disc & 1 & 1 & 1 \\
\hline Estazolam & N05CD04 & 1 & & & Disc & 1 & & 1 \\
\hline Famotidine & A02BA03 & 1 & & & Disc & 1 & 1 & 0 \\
\hline Fentanyl & N01AH01 & 1 & & 1 & 1 & 1 & 1 & 1 \\
\hline Fexofenadine & R06AX26 & 0 & & & 2 & 2 & 1 & 0 \\
\hline Flunitrazepam & N05CD03 & & & & Disc & & 1 & 1 \\
\hline Flupentixol & N05AF01 & & & & & & & 1 \\
\hline Fluoxetine & N06AB03 & 1 & & & 1 & 1 & 1 & 1 \\
\hline Flurazepam & N05CD01 & 1 & & & Disc & 1 & 1 & 1 \\
\hline Fluvoxamine & N06AB08 & 1 & & 1 & 1 & 1 & 1 & 1 \\
\hline Furosemide & C03CA01 & 1 & & 1 & Disc & 1 or 3 & 1 & 1 \\
\hline Gentamicin & J01GB03 & 1 & & & Disc & 1 & 1 & 0 \\
\hline Glycopyrronium inh & R03BB06 & & & & & & 1 & 2 \\
\hline Guaifenesin & R05CA03 & 0 & & & Disc & 1 & 1 & 1 \\
\hline Haloperidol & N05AD01 & 0 & 1 & 1 & 1 or 2 & 1 or 2 & 2 & 1 \\
\hline Hydralazine & C02DB02 & 1 & & 1 & Disc & 1 & 1 & 1 \\
\hline Hydrocodone & R05DA03 & 0 & & & 1 or 2 & 2 & & 1 \\
\hline Hydrocortisone & H02АB09 & 1 & & 1 & Disc & 1 & 1 & 1 \\
\hline Iloperidone & N05AX14 & & & & & 1 & & \\
\hline Ipratropium inh & R03BB01 & 0 & & & 3 & 3 & 1 & \\
\hline Isosorbide mononitrate & C01DA08 & 1 & & 1 & Disc & 1 & 1 & 0 \\
\hline Isosorbide dinitrate & C01DA14 & 1 & & 1 & Disc & 1 & 1 & 0 \\
\hline Ketorolac & M01AB15 & & & & 1 & 1 & 1 & 0 \\
\hline
\end{tabular}


Table 1. Cont.

\begin{tabular}{|c|c|c|c|c|c|c|c|c|}
\hline Drug Name & ATC Code & $\begin{array}{l}\text { Carnahan } \\
2006\end{array}$ & $\begin{array}{l}\text { Rudolph } \\
2008\end{array}$ & $\begin{array}{c}\text { Boustani } \\
2008\end{array}$ & $\begin{array}{c}\text { Durán } \\
2013\end{array}$ & $\begin{array}{l}\text { Salahudeen } \\
2015\end{array}$ & $\begin{array}{c}\text { Kiesel } \\
2018\end{array}$ & $\begin{array}{l}\text { Jun } \\
2019\end{array}$ \\
\hline Ketotifen & R06AX17 & 1 & & & Disc & 1 & & 1 \\
\hline Levocetirizine & R06AE09 & & & & & 1 & 1 & 1 \\
\hline Levodopa-carbidopa & N04BA02 & 0 & 1 & & Disc & 1 & 1 & 0 \\
\hline Lithium & N05AN01 & 0 & & & 1 & 1 & 1 & 0 \\
\hline Loperamide & A07DA03 & 1 & 2 & 1 & 1 or 2 & 1 or 2 & 2 & 1 \\
\hline Loratadine & R06AX13 & 0 & 2 & & 1 or 2 & 1 or 2 & 1 & 1 \\
\hline Lorazepam & N05BA06 & 1 & & & Disc & 1 & 1 & 1 \\
\hline Lumiracoxib & M01AH06 & & & & Disc & 1 & & \\
\hline Mebeverine & A03AA04 & & & & & & & 1 \\
\hline Metformin & A10BA02 & 0 & & & Disc & 1 & 1 & 0 \\
\hline Methocarbamol & M03BA03 & & 1 & & 1 & 1 & 1 & 1 \\
\hline Methotrexate & L04AX03 & 0 & & & Disc & 1 & 1 & 0 \\
\hline Methylprednisolone & H02AB04 & 1 & & & Disc & 1 & 1 & 0 \\
\hline Metoclopramide & A03FA01 & 0 & 1 & & Disc & 1 & 1 & 0 \\
\hline Metoprolol & C07AB02 & 0 & & 1 & 0 & 1 & 1 & 0 \\
\hline Midazolam & N05CD08 & 1 & & & Disc & 1 & 1 & 1 \\
\hline Mirtazapine & N06AX11 & 0 & 1 & & 1 & 1 & 1 & 1 \\
\hline Morphine & N02AA01 & 1 & & 1 & 1 & 1 & 1 & 1 \\
\hline Naratriptan & $\mathrm{N} 02 \mathrm{CC} 02$ & & & & Disc & 1 & 1 & 0 \\
\hline Nefazodone & N06AX06 & 0 & & & 1 & 1 & & \\
\hline Nifedipine & C08CA05 & 1 & & 1 & 0 & 1 & 1 & 0 \\
\hline Nizatidine & A02BA04 & 1 & & & Disc & 1 & & \\
\hline Oxazepam & N05BA04 & 1 & & & Disc & 1 & 1 & \\
\hline Oxycodone & N02AA05 & 1 & & & 1 & 1 & 1 & 1 \\
\hline Paliperidone & N05AX13 & & & & & 1 & 1 & 1 \\
\hline Pancuronium & M03AC01 & 1 & & & Disc & 1 & 1 & \\
\hline Phenelzine & N06AF03 & 1 & & & 1 & 1 & & \\
\hline Phenobarbital & N03AA02 & 0 & & & Disc & 1 & 1 & 0 \\
\hline Piperacillin & J01CA12 & 1 & & & Disc & 1 & 1 & 0 \\
\hline Pramipexole & N04BC05 & 0 & 1 & & Disc & 1 & 1 & \\
\hline Prednisolone & H02AB06 & 1 & & & Disc & 1 & 1 & 1 \\
\hline Prednisone & Н02АВ07 & 1 & & 1 & & 1 & 1 & \\
\hline Pridinol & М03ВХ03 & & & & & & & 1 \\
\hline Pseudoephedrine & R01BA02 & 0 & 2 & & Disc & 2 & 1 & 0 \\
\hline Quinidine & C01BA01 & 0 & & 1 & & 1 & 1 & \\
\hline Risperidone & N05AX08 & 0 & 1 & 1 & 1 & 1 & 1 & 1 \\
\hline Rotigotine ${ }^{\text {patch }}$ & N04BC09 & & & & & & 1 & \\
\hline Selegiline & N04BD01 & 0 & 1 & & Disc & 1 & 1 & 0 \\
\hline Sertraline & N06AB06 & 1 & & & 0 & 1 & 1 & 0 \\
\hline Sumatriptan & N02CC01 & & & & Disc & 1 & 1 & 0 \\
\hline Temazepam & N05CD07 & 1 & & & 1 & 1 & 1 & 1 \\
\hline Theophylline & R03DA04 & 1 & & 1 & 1 or 2 & 1 or 2 & 2 & 1 \\
\hline Tiotixene & N05AF04 & 1 & 3 & & 3 & 1 or 3 & & 1 \\
\hline Tiotropium inh & R03BB04 & & & & & & 1 & \\
\hline Trandolapril & C09AA10 & 0 & & & Disc & 1 & 1 & \\
\hline Trazodone & N06AX05 & 0 & 1 & 1 & 1 & 1 & 1 & 1 \\
\hline Triamcinolone & Н02АВ08 & 1 & & & Disc & 1 & 1 & 0 \\
\hline Triamterene & C03DB02 & 1 & & 1 & Disc & 1 & 1 & 0 \\
\hline Trimebutine & A03AA05 & & & & & & & 1 \\
\hline Triazolam & N05CD05 & 1 & & & 1 & 1 & 1 & 1 \\
\hline Valproic acid & N03AG01 & 1 & & & Disc & 1 & 1 & 0 \\
\hline Vancomycin & J01XA01 & 1 & & & Disc & 1 & 1 & 0 \\
\hline Venlafaxine & N06AX16 & 0 & & & 0 & 1 & 1 & 1 \\
\hline Warfarin & B01AA03 & 1 & & & 0 & 1 & 1 & 0 \\
\hline Ziprasidone & N05AE04 & & 1 & & Disc & 1 & 1 & 1 \\
\hline Zolmitriptan & N02CC03 & & & & Disc & 1 & 1 & 0 \\
\hline
\end{tabular}


Table 2. Medium potency anticholinergics (Score 2).

\begin{tabular}{|c|c|c|c|c|c|c|c|c|}
\hline Drug Name & ATC Code & $\begin{array}{c}\text { Carnahan } \\
2006\end{array}$ & $\begin{array}{l}\text { Rudolph } \\
2008\end{array}$ & $\begin{array}{c}\text { Boustani } \\
2008\end{array}$ & $\begin{array}{c}\text { Durán } \\
2013\end{array}$ & $\begin{array}{c}\text { Salahudeen } \\
2015\end{array}$ & $\begin{array}{c}\text { Kiesel } \\
2018\end{array}$ & $\begin{array}{l}\text { Jun } \\
2019\end{array}$ \\
\hline Amantadine & N04BB01 & 1 & 2 & 2 & 1 or 2 & 1 or 2 & 2 & 2 \\
\hline Baclofen & M03ВХ01 & 0 & 2 & & 2 & 2 & 1 & 1 \\
\hline Carbamazepine & N03AF01 & 2 & & 2 & 1 or 2 & 1 or 2 & 2 & 1 \\
\hline Cloperastine & R05DB21 & & & & & & & 2 \\
\hline Cimetidine & A02BA01 & 2 & 2 & 1 & 2 & 1 or 2 & 2 & 2 \\
\hline Cyclobenzaprine & M03BХ08 & 2 & 2 & 2 & 1 or 2 & 1 or 2 & & 2 \\
\hline Dosulepin & N06AA16 & & & & 2 & 2 & & \\
\hline Fluphenazine & N05AB02 & 1 & 3 & & 3 & 1 or 3 & 1 & \\
\hline Loxapine & N05AH01 & 2 & & 2 & 2 & 2 & 2 & 2 \\
\hline Maprotiline & N06AA21 & & & & Disc & 3 & 2 & \\
\hline Meperidine * & N02AB02 & 2 & & 2 & 2 & 2 & & 2 \\
\hline Methadone & N07BC02 & & & & 2 & 2 & 2 & \\
\hline Molindone & N05AE02 & 2 & & 2 & 2 & 2 & & 2 \\
\hline Nefopam & N02BG06 & & & & & 2 & & 2 \\
\hline Olanzapine & N05AH03 & 1 & 2 & 3 & 1 or 2 & 1,2 or 3 & 2 & 3 \\
\hline Oxcarbazepine & N03AF02 & 2 & & 2 & 2 & 2 & 2 & 2 \\
\hline Paroxetine & N06AB05 & 1 & 1 & 3 & 1 or 2 & 1,2 or 3 & 2 & 2 \\
\hline Perphenazine & N05AB03 & 1 & 3 & 3 & Disc & 1,2 or 3 & 1 & 2 \\
\hline Pimozide & N05AG02 & 2 & & 2 & 2 & 2 & 2 & 2 \\
\hline Prochlorperazine & N05AB04 & 1 & 2 & & 1 or 2 & 1 or 2 & & \\
\hline Promazine & N05AA03 & & & 3 & 2 & 2 & & \\
\hline Propoxyphene & N02AC04 & 0 & & & 1 or 2 & 2 & & \\
\hline Quetiapine & N05AH04 & 0 & 1 & 3 & 1 or 2 & 1,2 or 3 & 2 & 2 \\
\hline Ranitidine & A02BA02 & 2 & 1 & & 1 or 2 & 1 or 2 & 2 & 1 \\
\hline Tramadol & N02AX02 & 1 & & & 1 or 2 & 1 or 2 & 2 & 2 \\
\hline Triprolidine & R06AX07 & & & & & & & 2 \\
\hline Zotepine & N05AX11 & & & & & & & 2 \\
\hline Zuclopenthixol & N05AF05 & & & & & & & 2 \\
\hline
\end{tabular}

${ }^{*}$ Meperidine $/$ Pethidine. Disc $=$ discrepant for the authors.

Table 3. High potency anticholinergics (Score 3).

\begin{tabular}{|c|c|c|c|c|c|c|c|c|}
\hline Drug Name & ATC Code & $\begin{array}{c}\text { Carnahan } \\
2006\end{array}$ & $\begin{array}{l}\text { Rudolph } \\
2008\end{array}$ & $\begin{array}{c}\text { Boustani } \\
2008\end{array}$ & $\begin{array}{c}\text { Durán } \\
2013\end{array}$ & $\begin{array}{c}\text { Salahudeen } \\
2015\end{array}$ & $\begin{array}{c}\text { Kiesel } \\
2018\end{array}$ & $\begin{array}{l}\text { Jun } \\
2019\end{array}$ \\
\hline Acepromazine & N05AA04 & & & & 3 & 3 & & \\
\hline Amitriptyline & N06AA09 & 3 & 3 & 3 & 3 & 3 & 3 & 3 \\
\hline Amoxapine & N06AA17 & & & 3 & Disc & 3 & & 3 \\
\hline Atropine & A03BA01 & 3 & 3 & 3 & 3 & 3 & 3 & 3 \\
\hline Belladonna & A03BA04 & & & 2 & 3 & 2 or 3 & & 3 \\
\hline Benzatropine * & N04AC01 & 3 & 3 & 3 & 3 & 3 & & 3 \\
\hline Biperiden & N04AA02 & & & & & & & 3 \\
\hline Brompheniramine & R06AB01 & 3 & & 3 & 3 & 3 & & 3 \\
\hline Carbinoxamine & R06AA08 & 3 & & 3 & 3 & 3 & & 3 \\
\hline Carisoprodol & M03BA02 & 0 & 3 & & Disc & 3 & & \\
\hline Chlorphenamine * & R06AB04 & 3 & 3 & 3 & 3 & 3 & 3 & 3 \\
\hline Chlorpromazine & N05AA01 & 3 & 3 & 3 & 3 & 3 & & 3 \\
\hline Chlorprothixene & N05AF03 & & & & & & & 3 \\
\hline Cimetropium bromide & A03BB05 & & & & & & & 3 \\
\hline Clemastine & R06AA04 & 3 & & 3 & 3 & 3 & 3 & 3 \\
\hline Clomipramine & N06AA04 & 3 & & 3 & 3 & 3 & 3 & 3 \\
\hline Clozapine & N05AH02 & 3 & 2 & 3 & 3 & 2 or 3 & 3 & 3 \\
\hline Cyproheptadine & R06AX02 & 2 & 3 & 2 & 3 & 2 or 3 & 3 & 2 \\
\hline Darifenacin & G04BD10 & 3 & & 3 & 3 & 3 & 3 & \\
\hline Desipramine & N06AA01 & 3 & 2 & 3 & 3 & 2 or 3 & & \\
\hline Dexbrompheniramine & R06AB06 & & & & & & & 3 \\
\hline Dexchlorpheniramine & R06AB02 & & & & 3 & 3 & & 3 \\
\hline
\end{tabular}


Table 3. Cont.

\begin{tabular}{|c|c|c|c|c|c|c|c|c|}
\hline Drug Name & ATC Code & $\begin{array}{c}\text { Carnahan } \\
2006\end{array}$ & $\begin{array}{c}\text { Rudolph } \\
2008\end{array}$ & $\begin{array}{c}\text { Boustani } \\
2008\end{array}$ & $\begin{array}{c}\text { Durán } \\
2013\end{array}$ & $\begin{array}{c}\text { Salahudeen } \\
2015\end{array}$ & $\begin{array}{c}\text { Kiesel } \\
2018 \\
\end{array}$ & $\begin{array}{l}\text { Jun } \\
2019\end{array}$ \\
\hline Dicyclomine * & A03AA07 & 3 & 3 & 3 & 3 & 3 & & 3 \\
\hline Difemerine & A03AA09 & & & & & & & 3 \\
\hline Diphenhydramine * & R06AA02 & 3 & 3 & 3 & 3 & 3 & 3 & 3 \\
\hline Doxepin & N06AA12 & 3 & & 3 & 3 & 3 & 3 & 3 \\
\hline Doxylamine & R06AA09 & & & & & 3 & 1 & 3 \\
\hline Emepronium & G04BD01 & & & & 3 & 3 & & \\
\hline Fesoterodine & G04BD11 & & & & & 3 & 3 & \\
\hline Flavoxate & G04BD02 & 3 & & 3 & 3 & 3 & 3 & 3 \\
\hline Homatropine & S01FA05 & & & & 3 & 3 & & \\
\hline Hydroxyzine & N05BB01 & 3 & 3 & 3 & 3 & 3 & 3 & 3 \\
\hline Hyoscyamine & A03BA03 & 3 & 3 & 3 & 3 & 3 & & 3 \\
\hline Imipramine & N06AA02 & 3 & 3 & 3 & 3 & 3 & 3 & 3 \\
\hline Levomepromazine * & N05AA02 & 2 & & 2 & 3 & 3 & 3 & 2 \\
\hline Meclozine * & R06AE05 & 3 & 3 & 3 & 3 & 3 & & 3 \\
\hline Mequitazine & R06AD07 & & & & & & & 3 \\
\hline Nortriptyline & N06AA10 & 3 & 2 & 3 & 3 & 2 or 3 & 3 & 3 \\
\hline Opipramol & N06AA05 & & & & Disc & 3 & 2 & \\
\hline Orphenadrine & N04AB02 & 3 & & 3 & 3 & 3 & 3 & 3 \\
\hline Otilonium bromide & А03АВ06 & & & & & & & 3 \\
\hline Oxybutynin & G04BD04 & 3 & 3 & 3 & 3 & 2 or 3 & 3 & 3 \\
\hline Pheniramine & R06AB05 & & & & & & & 3 \\
\hline Procyclidine & N04AA04 & 3 & & 3 & 3 & 3 & 3 & 3 \\
\hline Promethazine & R06AD02 & 3 & 3 & 3 & 3 & 3 & 1 & \\
\hline Propantheline & A03AB05 & 3 & & 3 & 3 & 2 or 3 & & \\
\hline Propiverine & G04BD06 & & & & & 3 & 3 & 3 \\
\hline Protriptyline & N06AA11 & 3 & & & 3 & 3 & & \\
\hline Pyrilamine * & R03DA12 & 3 & & 3 & 3 & 3 & & 3 \\
\hline Scopolamine * & A04AD01 & 3 & 3 & 3 & 3 & 3 & 3 & 3 \\
\hline Solifenacin & G04BD08 & & & & & 3 & 3 & 3 \\
\hline Thioridazine & N05AC02 & 3 & 3 & 3 & 3 & 3 & 3 & 3 \\
\hline Tiemonium iodide & A03AB17 & & & & & & & 3 \\
\hline Timepidium bromide & A03AB19 & & & & & & & 3 \\
\hline Tizanidine & M03ВХ02 & & 3 & & 3 & 3 & 3 & 2 \\
\hline Tolterodine & G04BD07 & 3 & 2 & 3 & 3 & 2 or 3 & 3 & 3 \\
\hline Trifluoperazine & N05AB06 & 1 & 3 & 3 & Disc & 1 or 3 & & \\
\hline Trihexyphenidyl & N04AA01 & 3 & & 3 & 3 & 3 & 3 & 3 \\
\hline Trimipramine & N06AA06 & 3 & & 3 & 3 & 3 & 3 & \\
\hline Tropatepine & N04AA12 & & & & 3 & 3 & & \\
\hline Trospium & G04BD09 & & & & & 3 & 3 & 3 \\
\hline Valethamate & A03AX14 & & & & & & & 3 \\
\hline
\end{tabular}

* Benzatropine/ Benztropine; ${ }^{*}$ Dicyclomine/ Dicycloverine; ${ }^{*}$ Diphenhydramine/ Dimenhydrinate; ${ }^{*}$ Chlor phenamine/ Chlorpheniramine; ${ }^{*}$ Levomepromazine/ Methotrimeprazine; * Meclozine/ Meclizine; * Pyrilamine/ Mepyramine; $*$ Scopolamine $/$ Hyoscine. Disc $=$ discrepant for the authors.

Table 2 shows the 28 drugs with proposed medium potency (Score 2) selected for the CALS. In this case, two drugs were evaluated by the experts: disopyramide and prochlorperazine. Prochlorperazine was maintained with a score of 2 and disopyramide was changed to a score of 1 (Table 4).

Finally, the review selected 62 drugs with high anticholinergic potency (Score 3; Table 3). There were discrepancies in the score of six drugs rated as level 3: cyproheptadine, fluphenazine, maprotiline, olanzapine, paroxetine and perphenazine. All those assessed were changed to level 2 by the team's pharmacologists, except cyproheptadine, which remained at level 3. 
Table 4. CRIDECO Anticholinergic Load Scale.

\begin{tabular}{|c|c|c|c|c|c|c|}
\hline \multicolumn{4}{|c|}{ Low Potency (Score 1) } & \multirow{2}{*}{$\begin{array}{c}\begin{array}{c}\text { Medium } \\
\text { Potency } \\
\text { (Score 2) }\end{array} \\
\text { Amantadine }\end{array}$} & \multicolumn{2}{|c|}{ High Potency (Score 3) } \\
\hline Aclidinium $^{\text {inh }}$ & Cyclosporine & Iloperidone & Phenobarbital & & Acepromazine & Hyoscyamine \\
\hline Alimemazine * & Desloratadine & Ipratropium inh & Piperacillin & Baclofen & Amitriptyline & Imipramine \\
\hline Alprazolam & Desvelanfaxine & Isosorbide mononitrate & Pramipexole & Carbamazepine & Amoxapine & Levomepromazine * \\
\hline Alverine & Dexamethasone & Isosorbide $_{\text {dinitrate }}$ & Prednisolone & Cloperastine & Atropine & Meclozine * \\
\hline Amisulpride & Dextromethorphan & Ketorolac & Prednisone & Cimetidine & Belladonna & Mequitazine \\
\hline Ampicillin & Diazepam & Ketotifen & Pridinol & Cyclobenzaprin & Benzatropine ${ }^{*}$ & Nortriptyline \\
\hline Aripiprazole & Digitoxin & Levocetirizine & Pseudoephedrine & Dosulepin & Biperiden & Opipramol \\
\hline Asenapine & Digoxin & Levodopa-carbidopa & Quinidine & Fluphenazine & Brompheniramine & Orphenadrine \\
\hline Atenolol & Diltiazem & Lithium & Risperidone & Loxapine & Carbinoxamine & Otilonium bromide \\
\hline Azathioprine & Dipyridamole & Loperamide & Rotigotine patch & Maprotiline & Carisoprodol & Oxybutynin \\
\hline Benazepril & Disopyramide & Loratadine & Selegiline & Meperidine * & Chlorphenamine * & Pheniramine \\
\hline Betaxolol & Domperidone & Lorazepam & Sertraline & Methadone & Chlorpromazine & Procyclidine \\
\hline Bisacodyl & Entacapone & Lumiracoxib & Sumatriptan & Molindone & Chlorprothixene & Promethazine \\
\hline Bromocriptine & Escitalopram & Mebeverine & Tapentadol & Nefopam & Cimetropium bromide & Propantheline \\
\hline Bromperidol & Estazolam & Metformin & Temazepam & Olanzapine & Clemastine & Propiverine \\
\hline Bupropion & Famotidine & Methocarbamol & Theophylline & Oxcarbazepine & Clomipramine & Protriptyline \\
\hline Captopril & Fentanyl & Methotrexate & Tiotixene & Paroxetine & Clozapine & Pyrilamine * \\
\hline Cefamandole & Fexofenadine & Methylprednisolone & Tiotropium inh & Perphenazine & Cyproheptadine & Scopolamine * \\
\hline Cefoxitin & Flunitrazepam & Metoclopramide & Trandolapril & Pimozide & Darifenacin & Solifenacin \\
\hline Celecoxib & Flupentixol & Metoprolol & Trazodone & Prochlorperazine & Desipramine & Thioridazine \\
\hline Cephalothin & Fluoxetine & Midazolam & Triamcinolone & Promazine & Dexbrompheniramine & Tiemonium iodide \\
\hline Cetirizine & Flurazepam & Mirtazapine & Triamterene & Propoxyphene & Dexchlorpheniramine & Timepidium bromide \\
\hline Cinnarizine & Fluvoxamine & Morphine & Trimebutine & Quetiapine & Dicyclomine * & Tizanidine \\
\hline Chlordiazepoxide & Furosemide & Naratriptan & Triazolam & Ranitidine & Difemerine & Tolterodine \\
\hline Chlortalidone & Gentamicin & Nefazodone & Umeclidinium $^{\text {inh }}$ & Tramadol & Diphenhydramine * & Trifluoperazine \\
\hline Citalopram & Glycopyrronium inh & Nifedipine & Valproic acid & Triprolidine & Doxepin & Trihexyphenidyl \\
\hline Clindamycin & Guaifenesin & Nizatidine & Vancomycin & Zotepine & Doxylamine & Trimipramine \\
\hline Clonazepam & Haloperidol & Oxazepam & Venlafaxine & Zuclopenthixol & Emepronium & Tropatepine \\
\hline Clorazepate & Hydralazine & Oxycodone & Warfarin & & Fesoterodine & Trospium \\
\hline Codeine & Hydrocodone & Paliperidone & Ziprasidone & & Flavoxate & Valethamate \\
\hline Cortisone & Hydrocortisone & Pancuronium & Zolmitriptan & & Homatropine & \\
\hline Cycloserine & Hydromorphone & Phenelzine & & & Hydroxyzine & \\
\hline
\end{tabular}

Drugs in italics are not currently commercialized/authorized in Spain as of November 2021. * Alimemazine/ Trimeprazine; * Benzatropine/ Benztropine; * Dicyclomine/ dicycloverine; ${ }^{*}$ Diphenhydramine/ dimenhydrinate; ${ }^{*}$ Chlorphenamine/ chlorpheniramine; ${ }^{*}$ Levomepromazine/ Methotrimeprazine; ${ }^{*}$ Meclozine/ meclizine; * Meperidine/ Pethidine; ${ }^{*}$ Pyrilamine/ Mepyramine; ${ }^{*}$ Scopolamine/ Hyoscine. ${ }^{\text {inh }}=$ inhalative.

Table 4 shows the final scale, where medicines not commercialized or not authorized in Spain in November 2021 are shown in italics. The inhaled drug umeclidinium was added to this scale because the rest of the inhaled anticholinergic drugs were included (aclidinium, glycopyrronium, ipratropium and tiotropium) and it is also approved in Spain. The same applies to the drugs tapentadol and hydromorphone because of their pharmacological similarity to other opioids, which are included in Spanish anticholinergic drug bulletins. As in previous studies, the total anticholinergic burden (TAB) was obtained by adding the score of each drug. A score $\geq 3$ is considered as a clinically relevant anticholinergic burden $[42-44,47,48]$.

\subsection{Comparison with the ACB and CALS Scale in Individuals with Subjective Memory Complaints}

In this study, the participants $(n=512)$ were people aged between 50 and 96 with SMC. They were classified according to tests scores as suggestive of CI $(n=164,32.03 \%)$ or cognitively normal $(n=348,67.97 \%)$. Table 5 summarizes the demographic and clinical variables of the participants between both groups. Specifically, the TAB score was obtained for each patient on the new scale, as well as in the ACB scale, to compare its association with CI. 
Table 5. Characteristics of patients in the CRIDECO study.

\begin{tabular}{ccc}
\hline $\begin{array}{c}\text { Characteristics of Study Participants } \\
(\boldsymbol{n}=\mathbf{5 1 2})\end{array}$ & $\begin{array}{c}\text { Cognitive Decline } \\
(\boldsymbol{n}=\mathbf{1 6 4})\end{array}$ & $\begin{array}{c}\text { No Cognitive Decline } \\
(\boldsymbol{n}=\mathbf{3 4 8})\end{array}$ \\
\hline Age, years (mean (SD)) & $74.67(7.91)$ & $68.09(9.08)$ \\
Female $(n(\%))$ & $123(75 \%)$ & $255(73.27 \%)$ \\
BMI (mean (SD)) & $27.15(3.85)$ & $5.74(3.90)$ \\
Drugs (mean (SD)) & $6.52(3.31)$ & $0.87(1.37)$ \\
Mean TAB ACB score (mean (SD)) & $1.08(1.53)$ & $1.62(1.67)$ \\
Mean TAB CALS score (mean (SD)) & $2.14(1.88)$ & $86(24.71 \%)$ \\
TAB CALS score $\geq 3(n(\%))$ & $61(37.20 \%)$ & $49(14.10 \%)$ \\
TAB ACB score $\geq 3(n(\%))$ & $35(21.34 \%)$ & $7.09(0.99)$ \\
MIS test (mean (SD)) & $4.44(2.31)$ & $0.73(0.78)$ \\
SPMSQ test (mean (SD)) & $2.99(1.96)$ & $16.10(4.38)$ \\
SVF test (mean (SD)) & $10.05(3.82)$ & $77(22.13 \%)$ \\
Diabetes $(n(\%))$ & $40(24.39 \%)$ & $189(54.31 \%)$ \\
Hypertension $(n(\%))$ & $100(60.98 \%)$ & $159(45.69 \%)$ \\
Hypercholesterolemia $(n(\%))$ & $82(50 \%)$ & $100(28.74 \%)$ \\
Depression $(n(\%))$ & $62(37.80 \%)$ & $(28 \%)$ \\
\hline
\end{tabular}

$\mathrm{SD}=$ Standard Deviation; BMI = Body Mass Index; TAB ACB = Total Anticholinergic Burden measured by Anticholinergic Cognitive Burden Scale; TAB CALS = Total Anticholinergic Burden measured by CRIDECO Anticholinergic Load Scale; MIS = Memory Impairment Screen; SPMSQ = Short Portable Mental Questionnaire; SVF $=$ Semantic Verbal Fluency Test.

The mean TAB obtained with CALS for the non-cognitively impaired group was $1.62( \pm 1.67)$, while for the cognitively impaired group it was $2.14( \pm 1.88)$, showing statistically significant differences in the total anticholinergic burden between both groups $(p$-value $=0.0026)$ (Figure 3A). On the other hand, with the ACB scale no significant differences were observed ( $p$-value $=0.1439)$, the mean obtained for the group without CI was $0.87( \pm 1.37)$ and $1.08( \pm 1.53)$ for the group with CI (Figure 3B).

\section{A CRIDECO Anticholinergic Load Scale (CALS)}

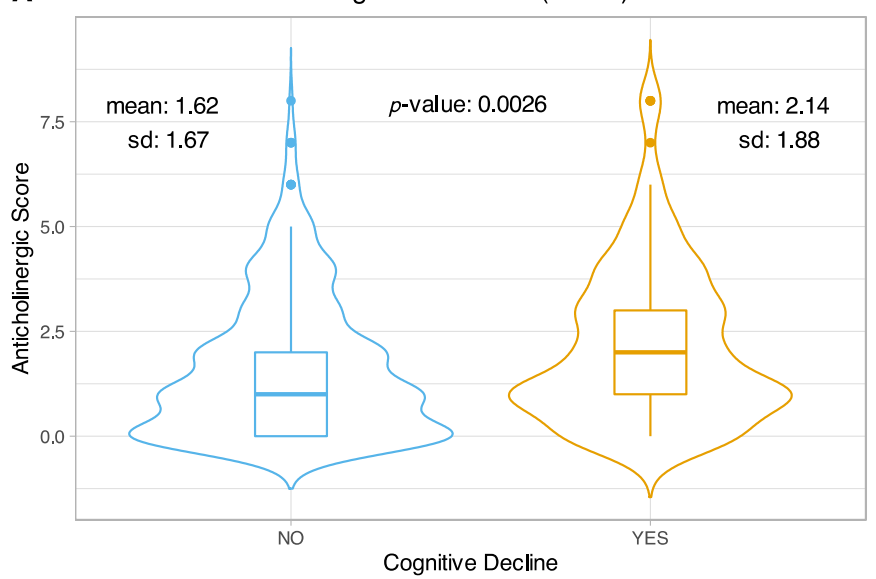

B Anticholinergic Cognitive Burden Scale (ACB)

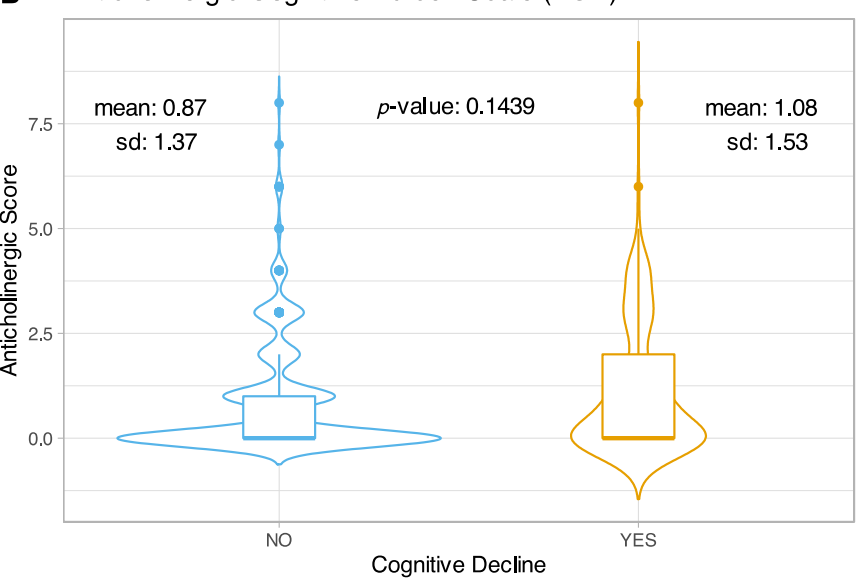

Figure 3. Violin plots comparing TAB scores in CI on the CALS (A) and ACB scales (B).

Next, we analyzed the relationship of the TAB values considered clinically relevant TAB $(\geq 3)$ and the presence of $C I$ in our population using the two scales (Figure 4). For clinically relevant TAB values, the CALS identified 148 individuals with a relevant anticholinergic score $(\geq 3)$ and 367 individuals with a lower anticholinergic score $(<3)$. Among patients with $\mathrm{CI}, 37 \%$ had a relevant TAB score, compared to $25 \%$ of patients without CI ( $p$-value $=0.005)$. On the other hand, with the ACB scale, $21 \%$ of patients with $\mathrm{CI}$ had a relevant TAB score, compared to $14 \%$ of those without cognitive impairment $(p$-value $=0.0521)$. 
A CRIDECO Anticholinergic Load Scale (CALS)

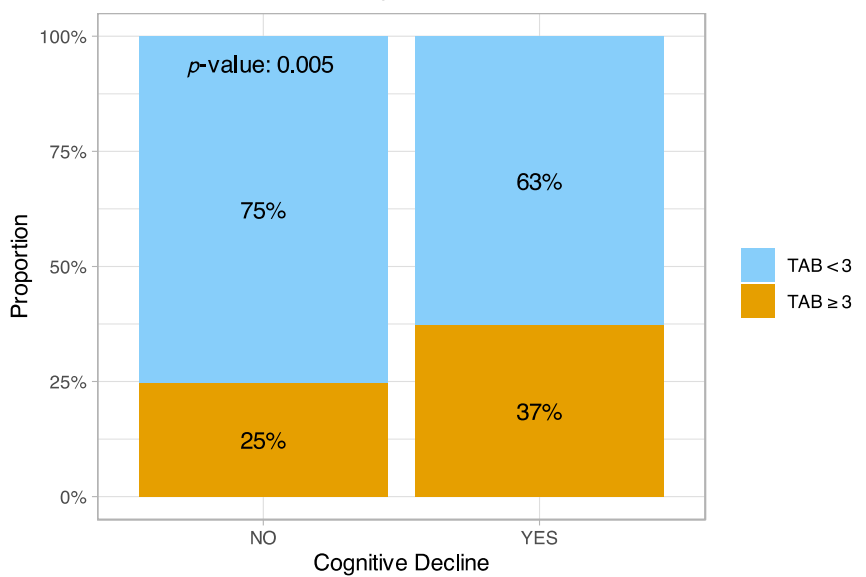

B Anticholinergic Cognitive Burden Scale (ACB)

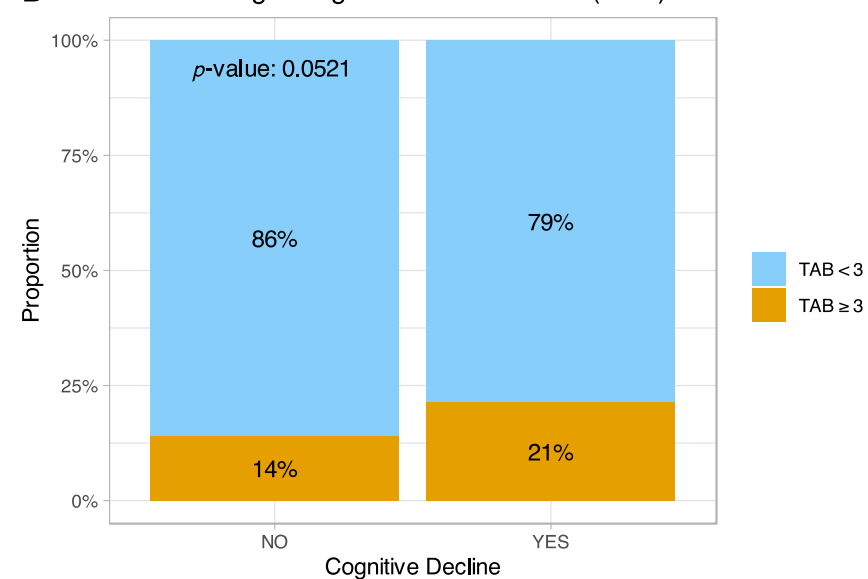

Figure 4. Graph comparing relevant anticholinergic burden and CI on the (A) CALS and (B) ACB scales. $\mathrm{TAB}=$ Total Anticholinergic Burden.

The fifteen most used drugs with anticholinergic effects in the study sample were obtained (Figure 5). These included four benzodiazepines, two long-acting (diazepam and clonazepam) and two intermediate-acting (lorazepam and alprazolam). Five antidepressants, including three selective serotonin reuptake inhibitors (SSRIs) (escitalopram, paroxetine, sertraline), a selective serotonin and norepinephrine reuptake inhibitor (SNRI) (desvenlafaxine) and a serotonin antagonist and reuptake inhibitor (SARI) (trazodone). A minor opioid alone (tramadol) and in combination with other analgesics (paracetamol). An oral antihyperglycemic drug alone (metformin) and in combination with other antidiabetics (sitagliptin and vildagliptin). And finally, a loop diuretic (furosemide).

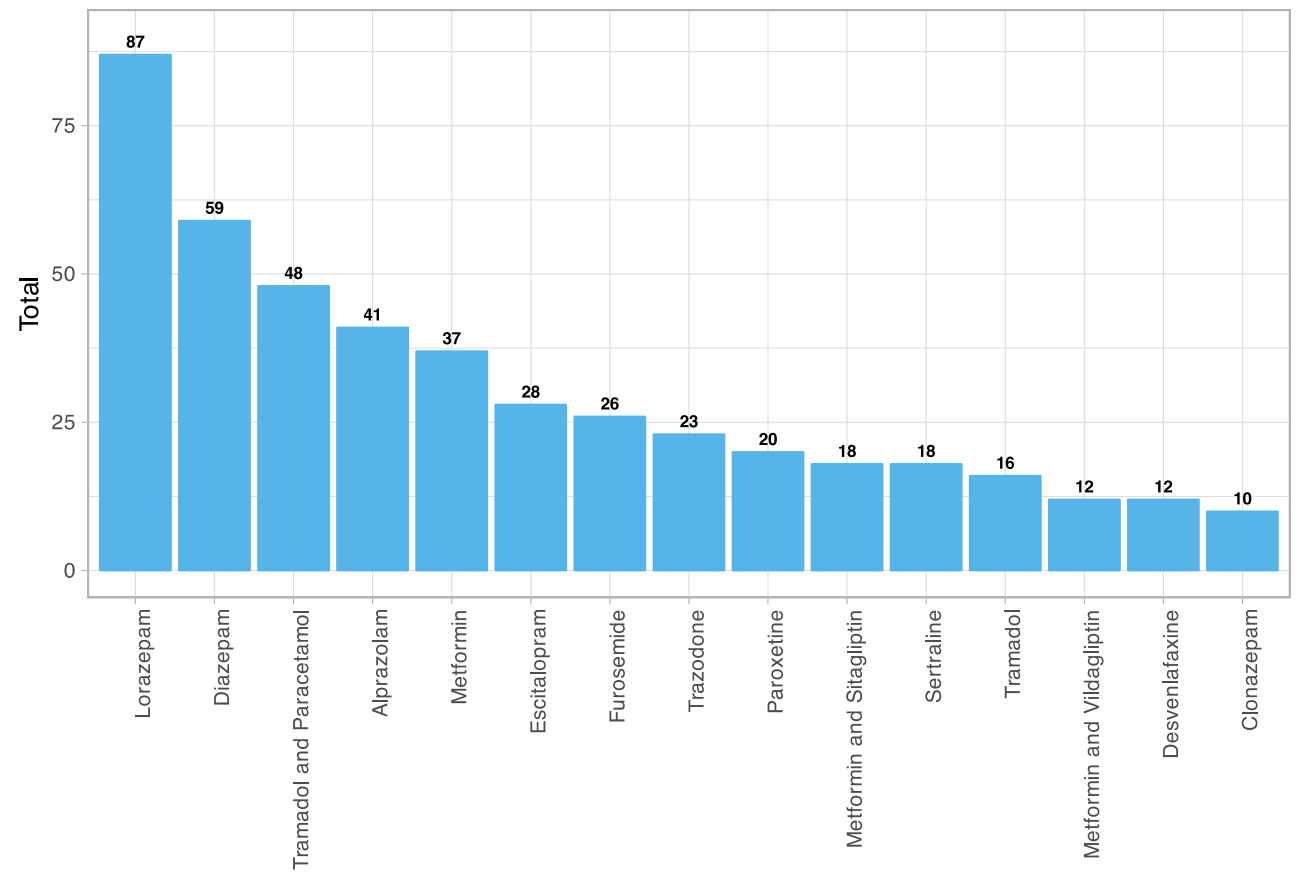

Figure 5. Anticholinergic drugs most used among patients with subjective memory complaints (CALS). N05BA06 = Lorazepam; N05BA01 = Diazepam; N02AJ13 = Tramadol and Paracetamol; N05BA12 = Alprazolam; A10BA02 = Metformin; N06AB10 = Escitalopram; C03CA01 = Furosemide; N06AX05 = Trazodone; N06AB05 = Paroxetine; A10BD07 = Metformin and Sitagliptin; N06AB06 = Sertraline; N02AX02 = Tramadol; A10BD08 = Metformin and Vildagliptin; N06AX23 = Desvenlafaxine; N03AE01 = Clonazepam. 
Furthermore, in our sample, the number of anticholinergic drugs used was significantly associated with CI-compatible scores in any of the three neuropsychological tests $(p$-value $=0.026)$. Specifically, the mean consumption in the group with CI was $6.52( \pm 3.31)$, compared to $5.74( \pm 3.28)$ in the group with a normal (or nonimpaired) score in all three tests.

Finally, a total of 38 patients were taking benzodiazepines (N05BA) and opioids (N02A), resulting in a central nervous system (CNS) depressant effect, which can lead to serious side effects such as slow or difficult breathing and death [63].

\section{Discussion}

Anticholinergic drugs form a heterogeneous group comprising active compounds with very diverse indications and applications. Many commonly used medications have anticholinergic effects. It is well known that antimuscarinic drugs can trigger both peripheral and central side effects such as the cognitive alterations that we observed in our population. Several longitudinal studies have linked exposure to various types of anticholinergics to increased risk of dementia [20-23]. Furthermore, the use of these drugs was associated with increased brain atrophy, dysfunction, and cognitive decline [64]. Similarly, there is a clear link between anticholinergic load and reduced cognitive performance [16-18]. Not only do these drugs have an impact on clinical entities such as CI, but they have also been linked to increased risk of falls [7] and mortality [24,25]. Furthermore, concomitant use of several medications with anticholinergic action will further increase the likelihood of adverse events [20]. However, a recent survey of pharmacists revealed that $45 \%$ of them did not know that these drugs were a risk factor for dementia [65] and only $44 \%$ of healthcare professionals knew that cognition was adversely affected by anticholinergics [66].

Low awareness of the risk of these drugs, together with the diversity of the anticholinergic burden scales, often makes them complex to identify by healthcare professionals. In the present work, we have tried to palliate this deficiency, collating the largest number of drugs with anticholinergic effects identified in the scientific literature. The newly developed scale not only includes drugs already published in previous studies, or authorized after those publications, but has also added three drugs authorized in Spain due to their pharmacological similarity to other drugs with reported anticholinergic effects. The aim is for it to serve as a clinical aid tool for the different health professionals, especially in our country.

Several scales have been designed and published over the years, but certain discrepancies remain among them. One of the main differences among the scales reported so far lies in the definition of whether a drug has an anticholinergic effect and also whether it has a high or low contribution to the global anticholinergic burden. These differences are one of the main sources of confusion when comparing scales [46]. At present, anticholinergic burden classification remains unclear, as the scores of different scales are based on subjective characteristics: bibliography and expert opinion. In addition, the wide variety of associated anticholinergic symptoms makes it difficult to assign a score from 0 to 3 . Despite the aforementioned limitations, a recent systematic review suggested that measuring anticholinergic activity based on a literature review was more effective in detecting significant adverse effects than measuring serum anticholinergic activity [67].

Moreover, several have been commercialized or withdrawn from the market by the different regulatory agencies over time. Thus, the updating of the different scales becomes crucial. Therefore, the development of an updated anticholinergic scale compiling as many drugs with anticholinergic activity as possible may be useful.

Our systematic review analysis selected seven anticholinergic scales, whose publication ranged from 2006 to 2019, with the ACB scale being the most widely used to date according to citation analyses [44]. In addition, a recent systematic review comparing 19 scales ranked the ACB and the GABS scale with the highest quality ratings [27]. In fact, these were the reasons why this scale was chosen for comparison with the CALS. 
To the best of our knowledge, this scale, with 217 active ingredients, currently includes the largest number of drugs. To minimize the selection bias that could appear by limiting the number of medicines in the scale, we added all the drugs obtained from the literature review, eliminating colchicine only, as two scales attributed no anticholinergic effects to it $[42,48]$ and two other manuscripts assessed it as discrepant $[45,46]$. With this new, more thorough and inclusive scale, we can calculate the total anticholinergic burden of a particular person and, depending on their general health situation, recommend alternatives to reduce the cholinergic burden.

When comparing two scales (CALS, ACB), we observed that the TAB obtained with the CALS was higher than that calculated with the ACB scale. Moreover, we observed a statistically significant association between either total TAB or clinically relevant TAB and CI, whereas no such associations were seen with the TABs derived from the ACB scale. This finding could be explained by the lower number of drugs with anticholinergic activity in the ACB scale compared to the CALS. We hypothesize that by not including some drugs with an anticholinergic effect recognized by numerous authors, their contribution to this cholinergic risk is lost in some patients.

It should be noted that the study sample is a special population, with patients with subjective memory complaints, so it is to be expected that the prevalence of dementia is higher than in the general population. In fact, the subjective memory complaint, in many patients, may be the first clinical manifestation after some AD histopathological hallmarks ( $\beta$-amyloid deposition, pathological tau, neurodegeneration), within a biological continuum in Alzheimer's disease [68]. Considering that anticholinergic drugs have been widely associated with cognitive decline, it is to be expected that a more sensitive scale will allow us to better discriminate the risk associated with this factor in a more specific population.

According to the cholinergic hypothesis, Alzheimer's disease is due to a decrease in acetylcholine synthesis [69]. Increasing acetylcholine levels by inhibiting acetylcholinesterase is one of the few therapeutic strategies to increase cognition and neural cell function [69]. Given that most of the drugs currently approved for the symptomatic treatment of Alzheimer's disease are aimed at restoring neuronal cholinergic activity [70], it would be contradictory to prescribe drugs whose pharmacological effect blocks this activity. Furthermore, the use of anticholinergic drugs in patients with dementia has been associated with increased mortality [25]. Therefore, in elderly patients, especially in patients with dementia [71] but also in individuals at risk such as those with subjective memory complaints [32], due to increased susceptibility to anticholinergic effects, the use of these drugs should be carefully evaluated.

Regarding the most prescribed anticholinergic drugs, benzodiazepines, antidepressants, hypoglycaemic drugs and opioids were the most consumed drugs in our population. This coincides with the fact that those same medications are also widely used among elderly patients. The fact that those drugs exhibit low anticholinergic activity (score $=1$ ) individually, except for tramadol and paroxetine (score $=2$ for both), reinforces the need for a standardized scale to alert clinicians to avoid co-prescription of these drugs when needed. Furthermore, tramadol is a drug that is not included in the ACB scale, being a widely established drug with an anticholinergic effect [42,45-48]. In Spain, this drug is extensively used for the treatment of chronic pain, so those patients taking it are only one point away from having a clinically relevant anticholinergic burden.

Niikawa and colleagues reported the existence of an association between polypharmacy and CI [72]. On the other hand, Baek and collaborators and Tapianen and colleagues linked increased consumption of benzodiazepines to cognitive decline [73,74]. This is consistent with our findings in our population, selected by reporting SMC, where an increase in the number and use of anticholinergic drugs prescribed, and the corresponding TAB score, correlated with the presence of CI in our neuropsychological tests. 


\section{Strengths and Limitations}

One potential limitation of the CALS is the variability of authorized anticholinergic drugs between countries and the impact this may have on an individual's final anticholinergic score. To avoid this limitation, we tried to include in this new scale most drugs with reported anticholinergic activity and we did not limit the scale to those compounds authorized in our country but collected information from several international scales, some frequently used in different studies.

Regarding the methodology of medication registration, one strength of the present study is that it was recorded through a personal interview and a review of the centralized electronic prescription registry of individuals. Therefore, drugs prescribed but probably not taken by patients were eliminated when applying the CALS. Because of this, the results obtained do reflect the actual patient's anticholinergic burden. Another aspect related to medication registration was the ATC code used. We decided to use it to classify active ingredients because it is the international code of drugs classification. Nevertheless, by using it, we identified a limitation when identifying with the same ATC a variable combination of active ingredients. However, this was resolved by taking these combinations as separate active ingredients taken simultaneously.

Another limitation of our study may be found in the specificity of the population under study. Although they all have high sensitivity and specificity for Alzheimer's disease, bear in mind that these parameters may vary depending on the origin of the $\mathrm{CI}$ and the clinical situation of the individual under study. Despite this, it is known from clinical practice that, if a patient has an alteration in any of these tests, it is highly probable that he or she has CI. In addition, these tests are also used in multiple studies as tools to measure CI. In any event, the neuropsychological screening performed does not yield a diagnosis but is an indication of the existence of cognitive problems. In our research project, individuals scoring as likely to have CI are directed to primary or tertiary health providers for full exploration and diagnosis.

\section{Conclusions}

We describe here a new anticholinergic scale that gathers information on 217 active principles, expanding previously existing scales. The new CALS was able to discriminate, more broadly than the ACB scale, the risk association between anticholinergic use and CI. Clinically relevant TAB scores measured with the CALS scale were associated with CI, so the presence of an updated tool in standard clinical practice may be useful, especially when prescribing medication to elderly patients with subjective memory complaints or with a dementia diagnosis. The development of updated scales may help provide us with a better measure of the influence of these drugs on neurodegenerative diseases such as dementia in longitudinal clinical studies. Nevertheless, further research is needed to validate the present scale and future updates will be required. Finally, with the use of this tool, clinicians can be more aware of their prescriptions and make a better personalized pharmacological approach for each patient.

Author Contributions: Conceptualization, H.R. and L.M.; methodology, H.R., L.M., J.P.-T. and J.P.; software, J.P.; validation, J.P.-T., C.C.-P. and G.G.-L.; formal analysis, J.P.; investigation, H.R., L.M., J.P.-T. and G.G.-L.; resources, L.M.; data curation, H.R. and J.P.; writing-original draft preparation, H.R. and G.G.-L.; writing-review and editing, L.M., J.P.-T., H.R., C.C.-P. and J.P.; visualization, J.P.; supervision, L.M., C.C.-P., J.P. and J.P.-T.; project administration, L.M.; funding acquisition, L.M. All authors have read and agreed to the published version of the manuscript.

Funding: This work was supported by SANTANDER-CEU FUSP-BS-PPC26/2018.

Institutional Review Board Statement: The study was approved by the Research Ethics Committee at the Universidad CEU Cardenal Herrera (approval no. CEI18/027, date of approval: 2 February 2018 and by the drugs Research Ethics Committee at Arnau de Vilanova Hospital (MOR-ROY-2018-013, date of approval: 18 July 2018). In accordance with the Declaration of Helsinki, all the participants gave their written informed consent to participate. 
Informed Consent Statement: Informed consent was obtained from all subjects involved in the study.

Data Availability Statement: The data used for this study is available upon request.

Acknowledgments: We would like to acknowledge all the pharmacies that participated in this study, all the general practitioners who collaborated in the diagnosis, Cátedra DeCo Micof-UCH who partially funded this research and all the patients who trusted us and participated in this work.

Conflicts of Interest: The authors declare no conflict of interest.

\section{References}

1. World Health Organization. Life Expectancy at Birth (Years). Available online: https://www.who.int/data/gho/data/indicators/ indicator-details/GHO/life-expectancy-at-birth-(years) (accessed on 12 November 2021).

2. Gutiérrez-Valencia, M.; Herce, P.A.; Lacalle-Fabo, E.; Escámez, B.C.; Cedeno-Veloz, B.; Martínez-Velilla, N. Prevalence of polypharmacy and associated factors in older adults in Spain: Data from the National Health Survey 2017. Med. Clin. 2019, 153, 141-150. [CrossRef] [PubMed]

3. Ahmed, B.; Nanji, K.; Mujeeb, R.; Patel, M.J. Effects of polypharmacy on adverse drug reactions among geriatric outpatients at a tertiary care Hospital in Karachi: A prospective cohort study. PLoS ONE 2014, 9, e112133. [CrossRef]

4. González-Bueno, J.; Sevilla-Sánchez, D.; Puigoriol-Juvanteny, E.; Molist-Brunet, N.; Codina-Jané, C.; Espaulella-Panicot, J. Factors associated with medication non-adherence among patients with multimorbidity and polypharmacy admitted to an intermediate care center. Int. J. Environ. Res. Public Health 2021, 18, 9606. [CrossRef] [PubMed]

5. Veronese, N.; Stubbs, B.; Noale, M.; Solmi, M.; Pilotto, A.; Vaona, A.; Demurtas, J.; Mueller, C.; Huntley, J.; Crepaldi, G.; et al. Polypharmacy Is Associated With Higher Frailty Risk in Older People: An 8-Year Longitudinal Cohort Study. J. Am. Med. Dir. Assoc. 2017, 18, 624-628. [CrossRef] [PubMed]

6. Park, H.Y.; Kim, S.; Sohn, H.S.; Kwon, J.W. The Association between Polypharmacy and Hip Fracture in Osteoporotic Women: A Nested Case-Control Study in South Korea. Clin. Drug Investig. 2019, 39, 63-71. [CrossRef]

7. Zaninotto, P.; Huang, Y.T.; Di Gessa, G.; Abell, J.; Lassale, C.; Steptoe, A. Polypharmacy is a risk factor for hospital admission due to a fall: Evidence from the English Longitudinal Study of Ageing. BMC Public Health 2020, 20, 1804. [CrossRef]

8. Grossi, C.M.; Richardson, K.; Savva, G.M.; Fox, C.; Arthur, A.; Loke, Y.K.; Steel, N.; Brayne, C.; Matthews, F.E.; Robinson, L.; et al. Increasing prevalence of anticholinergic medication use in older people in England over 20 years: Cognitive function and ageing study i and II. BMC Geriatr. 2020, 20, 267. [CrossRef]

9. Nishtala, P.S.; Salahudeen, M.S.; Hilmer, S.N. Anticholinergics: Theoretical and clinical overview. Expert Opin. Drug Saf. 2016, 15, 753-768. [CrossRef]

10. Tune, L.E. Anticholinergic effects of medication in elderly patients. J. Clin. Psychiatry 2001, 62, 11-14.

11. Chancellor, M.B.; Staskin, D.R.; Kay, G.G.; Sandage, B.W.; Oefelein, M.G.; Tsao, J.W. Blood-brain barrier permeation and efflux exclusion of anticholinergics used in the treatment of overactive bladder. Drugs Aging 2012, 29, 259-273. [CrossRef]

12. Gorup, E.; Rifel, J.; Petek Šter, M. Anticholinergic burden and most common anticholinergic-acting medicines in older general practice patients. Zdr. Varst. 2018, 57, 140-147. [CrossRef] [PubMed]

13. Best, O.; Gnjidic, D.; Hilmer, S.N.; Naganathan, V.; McLachlan, A.J. Investigating polypharmacy and drug burden index in hospitalised older people. Intern. Med. J. 2013, 43, 912-918. [CrossRef] [PubMed]

14. Sathienluckana, T.; Unaharassamee, W.; Suthisisang, C.; Suanchang, O.; Suansanae, T. Anticholinergic discontinuation and cognitive functions in patients with schizophrenia: A pharmacist-physician collaboration in the outpatient department. Integr. Pharm. Res. Pract. 2018, 7, 161-171. [CrossRef]

15. Bishara, D.; Harwood, D.; Sauer, J.; Taylor, D.M. Anticholinergic effect on cognition (AEC) of drugs commonly used in older people. Int. J. Geriatr. Psychiatry 2017, 32, 650-656. [CrossRef] [PubMed]

16. Pasina, L.; Lucca, U.; Tettamanti, M. Relation between anticholinergic burden and cognitive impairment: Results from the Monzino 80-plus population-based study. Pharmacoepidemiol. Drug Saf. 2020, 29, 1696-1702. [CrossRef] [PubMed]

17. Chatterjee, S.; Bali, V.; Carnahan, R.M.; Chen, H.; Johnson, M.L.; Aparasu, R.R. Anticholinergic burden and risk of cognitive impairment in elderly nursing home residents with depression. Res. Soc. Adm. Pharm. 2020, 16, 329-335. [CrossRef] [PubMed]

18. Sargent, L.; Nalls, M.; Amella, E.J.; Mueller, M.; Lageman, S.K.; Bandinelli, S.; Colpo, M.; Slattum, P.W.; Singleton, A.; Ferrucci, L. Anticholinergic Drug Induced Cognitive and Physical Impairment: Results from the InCHIANTI Study. J. Gerontol. A Biol. Sci. Med. Sci 2020, 75, 995-1002. [CrossRef] [PubMed]

19. Weigand, A.J.; Bondi, M.W.; Thomas, K.R.; Campbell, N.L.; Galasko, D.R.; Salmon, D.P.; Sewell, D.; Brewer, J.B.; Feldman, H.H.; Delano-Wood, L. Association of anticholinergic medications and AD biomarkers with incidence of MCI among cognitively normal older adults. Neurology 2020, 95, e2295-e2304. [CrossRef]

20. Gray, S.L.; Anderson, M.L.; Dublin, S.; Hanlon, J.T.; Hubbard, R.; Walker, R.; Yu, O.; Crane, P.K.; Larson, E.B. Cumulative use of strong anticholinergics and incident dementia: A prospective cohort study. JAMA Intern. Med. 2015, 175, 401-407. [CrossRef]

21. Richardson, K.; Fox, C.; Maidment, I.; Steel, N.; Loke, Y.K.; Arthur, A.; Myint, P.K.; Grossi, C.M.; Mattishent, K.; Bennett, K.; et al. Anticholinergic drugs and risk of dementia: Case-control study. BMJ 2018, 361, k1315. [CrossRef] 
22. Grossi, C.M.; Richardson, K.; Fox, C.; Maidment, I.; Steel, N.; Loke, Y.K.; Arthur, A.; Myint, P.K.; Campbell, N.; Boustani, M.; et al. Anticholinergic and benzodiazepine medication use and risk of incident dementia: A UK cohort study. BMC Geriatr. 2019, 19, 276. [CrossRef]

23. Coupland, C.A.C.; Hill, T.; Dening, T.; Morriss, R.; Moore, M.; Hippisley-Cox, J. Anticholinergic Drug Exposure and the Risk of Dementia: A Nested Case-Control Study. JAMA Intern. Med. 2019, 179, 1084-1093. [CrossRef] [PubMed]

24. Graves-Morris, K.; Stewart, C.; Soiza, R.L.; Taylor-Rowan, M.; Quinn, T.J.; Loke, Y.K.; Myint, P.K. The prognostic value of anticholinergic burden measures in relation to mortality in older individuals: A systematic review and meta-analysis. Front. Pharmacol. 2020, 11, 570. [CrossRef] [PubMed]

25. McMichael, A.J.; Zafeiridi, E.; Ryan, M.; Cunningham, E.L.; Passmore, A.P.; McGuinness, B. Anticholinergic drug use and risk of mortality for people with dementia in Northern Ireland. Aging Ment. Health 2021, 25, 1475-1482. [CrossRef]

26. Kalisch Ellett, L.M.; Pratt, N.L.; Ramsay, E.N.; Barratt, J.D.; Roughead, E.E. Multiple anticholinergic medication use and risk of hospital admission for confusion or dementia. J. Am. Geriatr. Soc. 2014, 62, 1916-1922. [CrossRef] [PubMed]

27. Lisibach, A.; Benelli, V.; Ceppi, M.G.; Waldner-Knogler, K.; Csajka, C.; Lutters, M. Quality of anticholinergic burden scales and their impact on clinical outcomes: A systematic review. Eur. J. Clin. Pharmacol. 2021, 77, 147-162. [CrossRef] [PubMed]

28. Jack, C.R.; Bennett, D.A.; Blennow, K.; Carrillo, M.C.; Dunn, B.; Haeberlein, S.B.; Holtzman, D.M.; Jagust, W.; Jessen, F.; Karlawish, J.; et al. NIA-AA Research Framework: Toward a biological definition of Alzheimer's disease. Alzheimer's Dement. 2018, 14, 535-562. [CrossRef]

29. Jessen, F.; Amariglio, R.E.; Buckley, R.F.; van der Flier, W.M.; Han, Y.; Molinuevo, J.L.; Rabin, L.; Rentz, D.M.; Rodriguez-Gomez, O.; Saykin, A.J.; et al. The characterisation of subjective cognitive decline. Lancet Neurol. 2020, 19, 271-278. [CrossRef]

30. Rabin, L.A.; Smart, C.M.; Amariglio, R.E. Subjective Cognitive Decline in Preclinical Alzheimer's Disease. Annu. Rev. Clin. Psychol. 2017, 13, 369-396. [CrossRef]

31. Jessen, F. Subjective and objective cognitive decline at the pre-dementia stage of Alzheimer's disease. Eur. Arch. Psychiatry Clin. Neurosci. 2014, 264, S3-S7. [CrossRef]

32. Mitchell, A.J.; Beaumont, H.; Ferguson, D.; Yadegarfar, M.; Stubbs, B. Risk of dementia and mild cognitive impairment in older people with subjective memory complaints: Meta-analysis. Acta Psychiatr. Scand. 2014, 130, 439-451. [CrossRef]

33. Liberati, A.; Altman, D.G.; Tetzlaff, J.; Mulrow, C.; Gøtzsche, P.C.; Ioannidis, J.P.A.; Clarke, M.; Devereaux, P.J.; Kleijnen, J.; Moher, D. The PRISMA statement for reporting systematic reviews and meta-analyses of studies that evaluate healthcare interventions: Explanation and elaboration. BMJ 2009, 339, b2700. [CrossRef]

34. Klamer, T.T.; Wauters, M.; Azermai, M.; Durán, C.; Christiaens, T.; Elseviers, M.; Vander Stichele, R. A Novel Scale Linking Potency and Dosage to Estimate Anticholinergic Exposure in Older Adults: The Muscarinic Acetylcholinergic Receptor ANTagonist Exposure Scale. Basic Clin. Pharmacol. Toxicol. 2017, 120, 582-590. [CrossRef] [PubMed]

35. Weichert, I.; Romero-Ortuno, R.; Tolonen, J.; Soe, T.; Lebus, C.; Choudhury, S.; Nadarajah, C.V.; Nanayakkara, P.; Orrù, M.; Di Somma, S. Anticholinergic medications in patients admitted with cognitive impairment or falls (AMiCI). The impact of hospital admission on anticholinergic cognitive medication burden. Results of a multicentre observational study. J. Clin. Pharm. Ther. 2018, 43, 682-694. [CrossRef]

36. López-Álvarez, J.; Sevilla-Llewellyn-Jones, J.; Agüera-Ortiz, L. Anticholinergic Drugs in Geriatric Psychopharmacology. Front. Neurosci. 2019, 13, 1309. [CrossRef]

37. Lee, M.S.; Kisely, S.; Zolotarev, B.; Warren, A.; Henderson, J.; George, M. Anticholinergic burden in older inpatients on psychotropic medication: Do we care? Australas. Psychiatry 2017, 25, 566-570. [CrossRef] [PubMed]

38. Williams, A.; Sera, L.; McPherson, M.L. Anticholinergic Burden in Hospice Patients With Dementia. Am. J. Hosp. Palliat. Med. 2019, 36, 222-227. [CrossRef]

39. Salahudeen, M.S.; Chyou, T.Y.; Nishtala, P.S. Serum anticholinergic activity and cognitive and functional adverse outcomes in older people: A systematic review and meta-analysis of the literature. PLoS ONE 2016, 11, e0151084. [CrossRef]

40. Lozano-Ortega, G.; Johnston, K.M.; Cheung, A.; Wagg, A.; Campbell, N.L.; Dmochowski, R.R.; Ng, D.B. A review of published anticholinergic scales and measures and their applicability in database analyses. Arch. Gerontol. Geriatr. 2020, $87,103885$. [CrossRef] [PubMed]

41. Nery, R.T.; Reis, A.M.M. Development of a Brazilian anticholinergic activity drug scale. Einstein 2019, 17, eAO4435. [CrossRef] [PubMed]

42. Carnahan, R.M.; Lund, B.C.; Perry, P.J.; Pollock, B.G.; Gulp, K.R. The anticholinergic drug scale as a measure of drug-related anticholinergic burden: Associations with serum anticholinergic activity. J. Clin. Pharmacol. 2006, 46, 1481-1486. [CrossRef] [PubMed]

43. Rudolph, J.L.; Salow, M.J.; Angelini, M.C.; McGlinchey, R.E. The anticholinergic risk scale and anticholinergic adverse effects in older persons. Arch. Intern. Med. 2008, 168, 508-513. [CrossRef] [PubMed]

44. Boustani, M.; Campbell, N.; Munger, S.; Maidment, I.; Fox, C. Impact of anticholinergics on the ageing brain: A review and practical application. Aging Health 2008, 4, 311-320. [CrossRef]

45. Durán, C.E.; Azermai, M.; Vander Stichele, R.H. Systematic review of anticholinergic risk scales in older adults. Eur. J. Clin. Pharmacol. 2013, 69, 1485-1496. [CrossRef]

46. Salahudeen, M.S.; Duffull, S.B.; Nishtala, P.S. Anticholinergic burden quantified by anticholinergic risk scales and adverse outcomes in older people: A systematic review. BMC Geriatr. 2015, 15, 31. [CrossRef] 
47. Kiesel, E.K.; Hopf, Y.M.; Drey, M. An anticholinergic burden score for German prescribers: Score development. BMC Geriatr. 2018, 18, 239. [CrossRef] [PubMed]

48. Jun, K.; Hwang, S.; Ah, Y.M.; Suh, Y.; Lee, J.Y. Development of an Anticholinergic Burden Scale specific for Korean older adults. Geriatr. Gerontol. Int. 2019, 19, 628-634. [CrossRef]

49. Base de Datos de medicamentos del Consejo General de Farmacéuticos (BotPlus 2.0). Available online: https://botplusweb. portalfarma.com (accessed on 20 November 2021).

50. Ramos, H.; Pardo, J.; Sánchez, R.; Puchades, E.; Pérez-Tur, J.; Navarro, A.; Moreno, L. Pharmacist-Physician Interprofessional Collaboration to Promote Early Detection of Cognitive Impairment: Increasing Diagnosis Rate. Front. Pharmacol. 2021, 12, 579489. [CrossRef]

51. Ramos, H.; Alacreu, M.; Guerrero, M.D.; Sánchez, R.; Moreno, L. Lifestyle Variables Such as Daily Internet Use as Promising Protective Factors against Cognitive Impairment in Patients with Subjective Memory Complaints. Preliminary Results. J. Pers. Med. 2021, 11, 1366. [CrossRef]

52. 52. WHO Collaborating Centre for Drug Statistics Methodology, ATC Classification Index with DDDs, 2022. Oslo, Norway 2021. Available online: https:/ /www.whocc.no/atc_ddd_index/ (accessed on 16 November 2021).

53. Buschke, H.; Kuslansky, G.; Katz, M.; Stewart, W.F.; Sliwinski, M.J.; Eckholdt, H.M.; Lipton, R.B. Screening for dementia with the Memory Impairment Screen. Neurology 1999, 52, 231-238. [CrossRef]

54. Böhm, P.; Peña-Casanova, J.; Gramunt, N.; Manero, R.M.; Terrón, C.; Quiñones Úbeda, S. Spanish version of the Memory Impairment Screen (MIS): Normative data and discriminant validity. Neurologia 2005, 20, 402-411.

55. Pfeiffer, E. A short portable mental status questionnaire for the assessment of organic brain deficit in elderly patients. J. Am. Geriatr. Soc. 1975, 23, 433-441. [CrossRef] [PubMed]

56. Martínez de la Iglesia, J.; Dueñas, R.; Onís, M.C.; Aguado, C.; Albert, C.; Luque, R. Spanish language adaptation and validation of the Pfeiffer's questionnaire (SPMSQ) to detect cognitive deterioration in people over 65 years of age. Med. Clin. 2001, 117, 129-134. [CrossRef]

57. Lopes, M.; Brucki, S.M.D.; Giampaoli, V.; Mansur, L.L. Semantic Verbal Fluency test in dementia: Preliminary retrospective analysis. Dement. Neuropsychol. 2009, 3, 315-320. [CrossRef] [PubMed]

58. Canning, S.J.; Leach, L.; Stuss, D.; Ngo, L.; Black, S.E. Diagnostic utility of abbreviated fluency measures in Alzheimer disease and vascular dementia. Neurology 2004, 62, 556-562. [CrossRef]

59. Pardo, C.C.; González, A.L. The utility of the semantic verbal fluency test in diagnosis of dementia. Rev. Neurol. 1999, 29, 709-714. [CrossRef]

60. Muñoz-Almaraz, F.J.; Climent, M.T.; Guerrero, M.D.; Moreno, L.; Pardo, J. A machine learning approach to design an efficient selective screening of mild cognitive impairment. J. Vis. Exp. 2020, 155, e59649. [CrossRef] [PubMed]

61. RStudio Team. RStudio: Integrated Development for R. RStudio, PBC, Boston, MA. 2020. Available online: https:/ / www.rstudio. com (accessed on 28 November 2021).

62. Faul, F.; Erdfelder, E.; Buchner, A.; Lang, A.G. Statistical power analyses using G*Power 3.1: Tests for correlation and regression analyses. Behav. Res. Methods 2009, 41, 1149-1160. [CrossRef]

63. FDA U.S. Food and Drug Administration. Drug Safety Communications. Available online: https://www.fda.gov/media/99761/ download\#: \{\}:text=\%5B8\%2D31\%2D2016\%5D,or\%20difficult\%20breathing\%20and\%20deaths (accessed on 30 November 2021).

64. Risacher, S.L.; McDonald, B.C.; Tallman, E.F.; West, J.D.; Farlow, M.R.; Unverzagt, F.W.; Gao, S.; Boustani, M.; Crane, P.K.; Petersen, R.C.; et al. Association Between Anticholinergic Medication Use and Cognition, Brain Metabolism, and Brain Atrophy in Cognitively Normal Older Adults. JAMA Neurol. 2016, 73, 721-732. [CrossRef]

65. Ramos, H.; Moreno, L.; Gil, M.; García-Lluch, G.; Sendra-Lillo, J.; Alacreu, M. Pharmacists' Knowledge of Factors Associated with Dementia: The A-to-Z Dementia Knowledge List. Int. J. Environ. Res. Public Health 2021, 18, 9934. [CrossRef]

66. Soundararajan, K.; Balchandra, P. Staff Awareness of Anti-Cholinergic Burden (ACB)-A Qualitative Cross-Sectional Study in a Tertiary Care Hospital. Cureus 2021, 13, e14141. [CrossRef] [PubMed]

67. Lampela, P.; Lavikainen, P.; Garcia-Horsman, J.A.; Bell, J.S.; Huupponen, R.; Hartikainen, S. Anticholinergic drug use, serum anticholinergic activity, and adverse drug events among older people: A population-based study. Drugs Aging 2013, 30, 321-330. [CrossRef]

68. Martorana, A.; Assogna, M.; De Lucia, V.; Motta, C.; Bonomi, C.G.; Bernocchi, F.; Di Donna, M.G.; Koch, G. Cognitive reserve and Alzheimer's biological continuum: Clues for prediction and prevention of dementia. Minerva Med. 2021, 112, 441-447. [CrossRef]

69. Breijyeh, Z.; Karaman, R. Comprehensive Review on Alzheimer's Disease: Causes and Treatment. Molecules 2020, $25,5789$. [CrossRef] [PubMed]

70. Sharma, K. Cholinesterase inhibitors as Alzheimer's therapeutics (Review). Mol. Med. Rep. 2019, 20, 1479-1487. [CrossRef] [PubMed]

71. Montastruc, F.; Benevent, J.; Touafchia, A.; Chebane, L.; Araujo, M.; Guitton-Bondon, E.; Durrieu, G.; Arbus, C.; Schmitt, L.; Begaud, B.; et al. Atropinic (anticholinergic) burden in antipsychotic-treated patients. Fundam. Clin. Pharmacol. 2018, 32, 114-119. [CrossRef]

72. Niikawa, H.; Okamura, T.; Ito, K.; Ura, C.; Miyamae, F.; Sakuma, N.; Ijuin, M.; Inagaki, H.; Sugiyama, M.; Awata, S. Association between polypharmacy and cognitive impairment in an elderly Japanese population residing in an urban community. Geriatr. Gerontol. Int. 2017, 17, 1286-1293. [CrossRef] 
73. Baek, Y.H.; Kim, H.J.; Bae, J.H.; Lee, H.; Oh, I.S.; Kim, W.J.; Shin, J.Y. Benzodiazepine-Related Cognitive Impairment or Dementia: A Signal Detection Study Using a Case/Non-Case Approach. Psychiatry Investig. 2020, 17, 587-595. [CrossRef]

74. Tapiainen, V.; Taipale, H.; Tanskanen, A.; Tiihonen, J.; Hartikainen, S.; Tolppanen, A.M. The risk of Alzheimer's disease associated with benzodiazepines and related drugs: Aa nested case-control study. Acta Psychiatr. Scand. 2018, 138, 91-100. [CrossRef] 